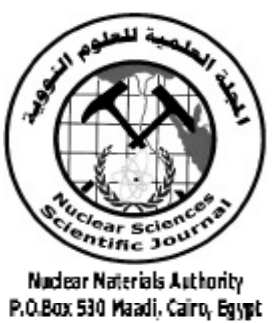

ISSN 2314-5609

Nuclear Sciences Scientific Journal

$8,141-171$

2019

http://www.ssnma.com

\title{
GEOLOGY, GEOCHEMISTERY AND RADIOACTIVITY OF POST COLLISION GRANITE AT WADI MURRA AREA, SOUTH EASTERN DESERT, EGYPT
}

\author{
ADEL H. EL AFANDY; KHAIRYA M. FAWZY'; HASSAN M. ASSRAN; EHAB K. \\ ABU ZEID and JEHAN B. EL SHAIEB \\ Nuclear Materials Authority, Cairo, Egypt ; ${ }^{1}$ Faculty of Sience, Aswan Univesity
}

\begin{abstract}
Wadi Murra area lies between Lat. 22 $24^{\prime}$ and $22^{\circ} 30^{\prime} \mathrm{N}$ and Long. $33^{\circ} 43^{\prime}$ and $33^{\circ} 52^{\prime}$ E. The studied younger granites in Wadi Murra area are represented by monzogranite, syenogranite and alkali feldspar granite. Wadi Murra granites have a semi rounded-NS oval shape $(11 \mathrm{x} 8 \mathrm{~km})$ comprising monzo and syenogranite in the middle grading to alkali-feldspar granite at the peripheries. Alkali feldspar granite intrudes the surrounding island arc metavolcanics as well as monzogranite of Gabal Umm Ara.

Monzo and syenogranite consist mainly of potash feldspar, plagioclase, quartz and mafic minerals with noticeable amount of opaque minerals. The main characteristic texture is equigranular with medium to coarse grain size. Porphyritic texture is also present. Alkali-feldspar granite is medium grained and characterized by hypidiomorphic granular texture and composed mainly of alkali-feldspar (orthoclase and minor microcline), quartz and subordinate plagioclase. The main mafic minerals are either amphibole or biotite. Accessories are represented by zircon, sphene, apatite and iron oxides.

Geochemically, the studied granites are calc-alkaline in nature, emplaced in post collision granite environment and have $\mathrm{A}_{2}$ character. The radiometric data for monzogranite show a wide variation in $\mathrm{eU}$ and eTh contents. The eU content ranges from 6 to $12 \mathrm{ppm}$ with an average of $8.5 \mathrm{ppm}$ and the eTh content ranges from 5 to $16 \mathrm{ppm}$ with an average of $10.12 \mathrm{ppm}$. The potassium content ranges between $3.08 \%$ and $7.67 \%$ with an average of $4.49 \%$. The eU content in syenogranite ranges from 2.4 to $6 \mathrm{ppm}$ with an average of $3.78 \mathrm{ppm}$ and the eTh content ranges from 7 to $9 \mathrm{ppm}$ with an average of $8 \mathrm{ppm}$. The potassium content is ranging between $4.08 \%$ and $4.69 \%$ with an average of $4.53 \%$. The eU content in alkali feldspar granite ranges from 5 to $9.8 \mathrm{ppm}$ with an average of $6.86 \mathrm{ppm}$ and the eTh content ranges from 8 to $16 \mathrm{ppm}$ with an average of $13.25 \mathrm{ppm}$. The potassium content is ranging between $4.9 \%$ and $4.95 \%$ with an average of $4.70 \%$.

The picked minerals from the anomalous samples are identified confirmed by environmental Scan Electron Microscope (ESEM) and XRD techniques at Laboratories of Nuclear Materials Authority (NMA). The identified minerals are classified to: 1) Secondary uranium minerals (uranophane, autunite and metautunite), 2) Thorium minerals (uranothorie), 3) Niobium-tantalum minerals (columbite), 4) Base metals minerals (pyrite) and 5) Accessory minerals bearing U, Th and REEs (zircon, fluorite, monazite, xenotime, sphene, allanite, apatite and iron oxides).
\end{abstract}




\section{INTRODUCTION}

The area of study is located in the southern part of the Eastern Desert at about $250 \mathrm{~km}$ southeast Aswan city. It is located north to Wadi Allaqi covering about $530 \mathrm{~km}^{2}$ and delineated by long. $33^{\circ} 43^{\prime}-33^{\circ} 52^{\prime} \mathrm{E}$ and lat. $22^{\circ}$ $24^{\prime}-22^{\circ} 30^{\prime} \mathrm{N}$.

A-type granitic rocks have drawn the attention of many workers in the last decades, but their origin is still subject of debate. There are three main models to explain the source of A-type granite magma: (1) Melting of felsic crust (e.g., Anderson, 1983; Clemens et al., 1986; Collins et al., 1982; Creaser et al., 1991); (2) Derivation from a mantle-derived mafic magma (e.g., Turner et al., 1992; Litvinovsky et al., 2002; Mushkin et al., 2003; Vander Auwera et al., 2003) and (3) Mixing of crustal and mantle sources (e.g., Barker et al., 1975; Foland and Allen, 1991; Frost and Frost, 1997). Despite intensive research, no consensus exists also on the origin of A-type magmatism in the Arabian-Nubian Shield (ANS).

The Arabian-Nubian Shield consists of Neoproterozoic juvenile crust formed by protracted accretion of island-arc terrains between 850 and $550 \mathrm{Ma}$ in the framework of the Gondwana supercontinent assembly (Stern, 1994; 2002 \& 2008; Nehlig et al., 2002; Stern and Johnson, 2010). The evolution of the ANS records three main tectonic stages of intra-oceanic subduction (850-700 Ma), collision and terrain amalgamation (700 to 635 $\mathrm{Ma})$, and tectonic escape, strike-slip faulting and extension (635 to $550 \mathrm{Ma}$ ) of the newly formed continental crust (Genna et al., 2002; Johnson and Woldehaimanot, 2003; Be'eriShlevin et al., 2009; Eyal et al., 2010; Stern and Johnson, 2010; Johnson et al., 2011). Post-collisional calc-alkaline to alkaline Atype granites (e.g., Ali et al., 2009 \& 2012; Be'eri-Shlevin et al., 2009b; Eyal et al., 2010; Moreno et al., 2012, Moghazi et al. 2012) intrude older post-collisional K-rich calc-alkaline I-type granitic rocks (Johnson, 2003; Eyal et al., 2010).
Different models have been proposed for the sources of the post-collisional A-type granites of the Arabian-Nubian Shield which invoke contrasting continental crust and mantle reservoirs (e.g., Katzir et al., 2007; Ali et al., 2009 \& 2014; Be'eri-Shlevin et al., 2009a \& 2010; El-Bialy and Hassen, 2012; Eyal et al., 2010; Farahat and Azer, 2011; Moghazi et al., 2011\&2012). These models are not conclusive because none of them could assess the crustal versus mantle contributions to explain the radiogenic isotopes and the juvenile character of the A-type magmatism (e.g., Eyal et al., 2010; Moreno et al.,2014).

Some of the post-collisional A-type granite plutons in the ANS are considered as specialized granites (Drysdall et al., 1984; Küster, 2009; Johnson et al., 2011). They are characterized by a marked enrichment in granitophile trace elements and valuable metals of economic interest, like $\mathrm{Nb}, \mathrm{Ta}, \mathrm{Zr}$, Th, U, Y, $\mathrm{Sn}$ and rare earth elements (REE).

Three different processes have been suggested to explain the rare metals endowments in the granitic rocks: (1) Enrichment controlled by magmatic processes (i.e. fractional crystallization), (Christiansen et al., 1984; 1986; Lehmann, 1982 \& 1987; Pollard et al., 1987; Rainbault, 1991; Cuney et al. 1992), (2) Volatile-phase transfer and complexation of rare metal elements and REE with $\mathrm{F}, \mathrm{Cl}, \mathrm{CO}_{2}$ (London, 1986 a\& b; Webster and Holloway, 1988; Salvi and Williams-Jones, 2005; Audétat et al., 2000; Webster et al., 2004; Schönenberger et al., 2008; Agangi et al., 2010), (3) Transportation of ore elements by hydrothermal aqueous fluids (Charoy and Pollard, 1989; Mass et al., 1987; Oreskes and Einaudi, 1990; Linnen and Cuney, 2005).

The aim of this study is to 1) Explain the relationship between the rare metal endowments and the hosting A-type granite complex; 2) Clarify the relationship between syenogranites and the alkali feldspars granites, which represent different suites of the A-type post-collisional magmatism and 3) Define the sources of the A-type granitic rocks. 


\section{GEOLOGY}

Wadi Murra granites are classified to older and younger granites. The older granites cropout at the northern and southeastern parts of the study standing as low mountainous elongate masses constituting about $101 \mathrm{~km}^{2}$, trending ENE-WSW and NNE-SSW and traversed by vast sandy plains. They also occur as small offshoots in the metasediments (Fig. 1). They are medium- to coarse-grained rocks ranging in colours from whitish grey, pinkish grey to grey characterized by intense weathering and exfoliation. Most of these granites are highly fractured and jointed. They could be distinguished to quartz diorite located in the south eastern part of the study area measuring about $36 \mathrm{~km}^{2}$. This rock is mostly weathered, although some small exposures are still fresh and granodiorite covers the northern part of the area of study and extends towards the ENE and NNE directions and characterized by low-leveled topography measuring of about $65 \mathrm{~km}^{2}$. The younger granites -cover an area of about $300 \mathrm{~km}^{2}$, characterized by moderate to high topography. They comprise monzogranites, syenogranites and alkali feldspar granites. All the granitic masses belong to the post-orogenic younger granite magmatic activity that intruded the Egyptian shield between 620 and $530 \mathrm{Ma}$ (Abdalla et al., 1996). They are epizonal and unfoliated masses that have sharp contacts with the surrounding country rocks or capped by the metavolcanics. The younger granites in the Wadi Murra area are sheared and highly jointed.

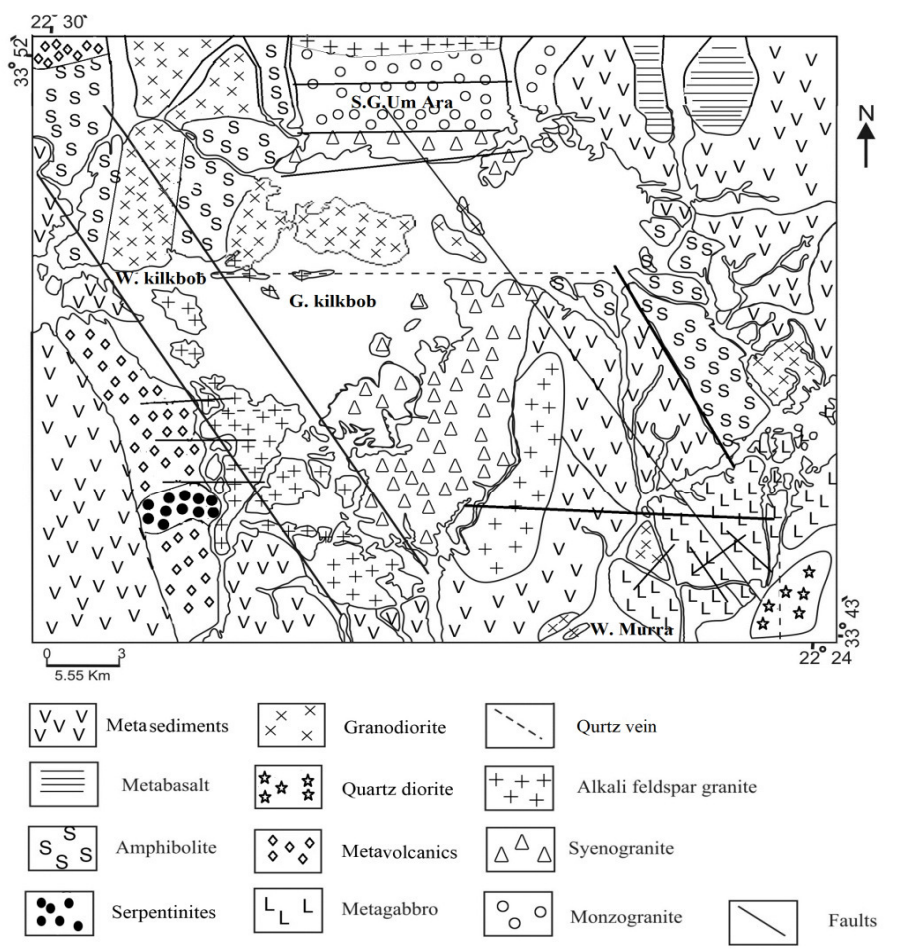

Fig.1:Geologic Map of Gabal Kilkabob Area (Modified after Nasr \& El-Sherbini, 2001) 


\section{Monzogranite}

It occurs as oval-shaped pluton trending $\mathrm{N}-\mathrm{S}$. It is generally of homogenous pinkish equigranular coarse-grained texture, but in places a porphiritic variety is recorded. It intruded by the syenogranite in the eastern side of G. Kilkbob. The weathered parts are characterized by reddish brown colour on surfaces and are hardly distinguished from the alkali feldspar granitoids (El-Gaby et al. 1988). Monzogranite has small xenoliths of metasediments and metavolcanics.

\section{Syenogranite}

It is the predominant rock variety in the area of study and follows the alkali-feldspar granite in relief and characterized by porphyritic texture. It is characterized by presence of uranium-bearing pegmatites occurring as dykes and lenticular bodies of about 15 meters in length and 1 meter in width. Some metasomatic alterations (Na-metasomatism) are observed in the syenogranites but intensively recorded in the alkali feldspar granite .

\section{Alkali Feldspar Granite}

It represents the main body of Gabal Kilkbob (570 meters above sea level) sending off shoots in metavolcanics. It is pink in colour and medium-grained characterized by porphyritic texture. Their contact with the Monzogranite is sharp and locally chilled. Pegmatites occur mainly along the contact zones as small dykes and knots.

\section{PETROGRAPHY}

\section{Monzogranite}

This granite is medium-grained characterized by pink to greyish pink colours and equigranular texture consisting of potash feldspars, plagioclase, quartz, mica as essential minerals, while the accessory minerals are represented by allanite, zircon, epidote, fluroapatite and sphene. Potash feldsparsar is the main feldspar representing about $34.5 \%$ of the rock and occurring as medium-grain crys- tals measured about $2.8 \mathrm{~mm}$. They are present as subhedral crystals of string perthite (Fig.2) and as anhedral crystals of antiperthite enclosing fine crystals of albite (Fig.3). The antiperthitic crystal is also formed as the potash feldspar invades and corrodes plagioclase (Fig.4). Plagioclase $\left(\mathrm{An}_{16}\right)$ is less dominant representing about $30.9 \%$ of the rock. It occurs as euhedral zoned crystals with Carlsbad twinning (Fig.4). Subhedral crystals are present as blade-like crystals of oligoclase characterized by lamellar twining and associated with myrmekitic texture where vermicular quartz is present (Fig.5). Some crystals of plagioclase are cracked and hematitized (Fig.4).

Feldspars are also present as disordered crystal composed of lamellar and crosshatched twinning where the microcline is transformed to albite (Deer et al., 1985). Quartz represents about $30.4 \%$ of the rock constituents occurring as anhedral crystals associating the other constituents (Figs.4-6) or as coarse crystals (up to $5.0 \mathrm{~mm}$ ) enclosing finer crystals of albite and disordered feldspars (Fig.7).

Mica is present as biotite and muscovite or chlorite as an alteration product of biotite. The mafics in monzogranite representing about $2.3 \%$ of the rock. It occurs as platy flakes of biotite (as primary mica mineral) character-

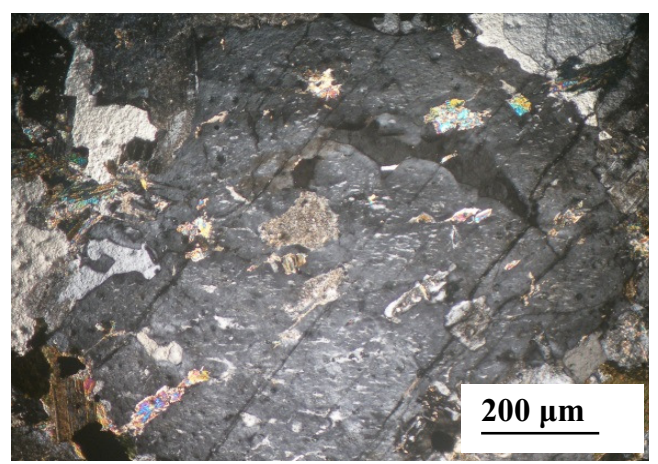

Fig.2:Photomicrograph for monzogranite of Wadi Murra area showing Potash feldspars as subhedral crystals of string perthite, XPL. 


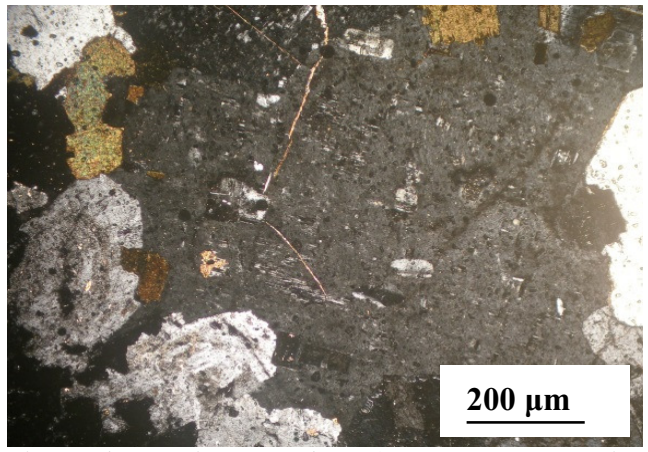

Fig.3:Photomicrograph for monzogranite of Wadi Murra area showing crystals of antiperthite enclosing very fine crystals of albite, XPL

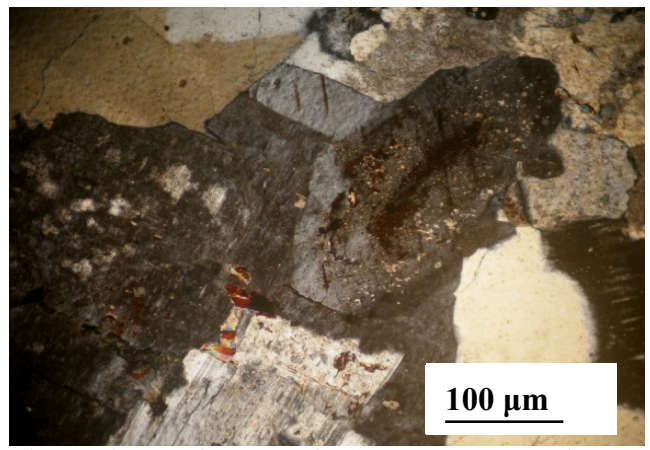

Fig.4:Photomicrograph for monzogranite of Wadi Murra area showing Antiperthitic crystal invades and corrodes plagioclase, XPL

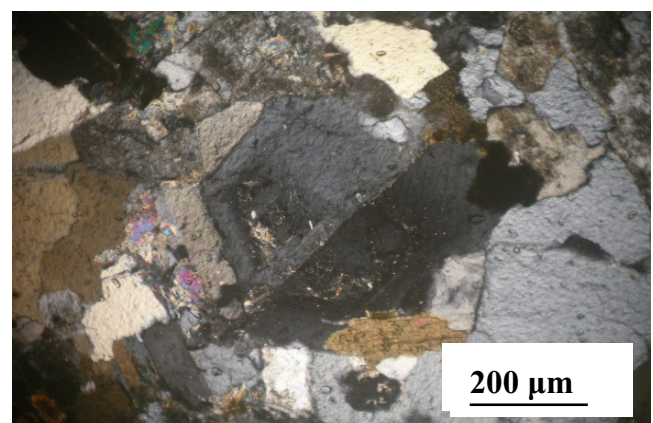

Fig.5:Photomicrograph for monzogranite of Wadi Murra area showing blade-like crystals of oligoclase showing lamellar twinning, XPL.

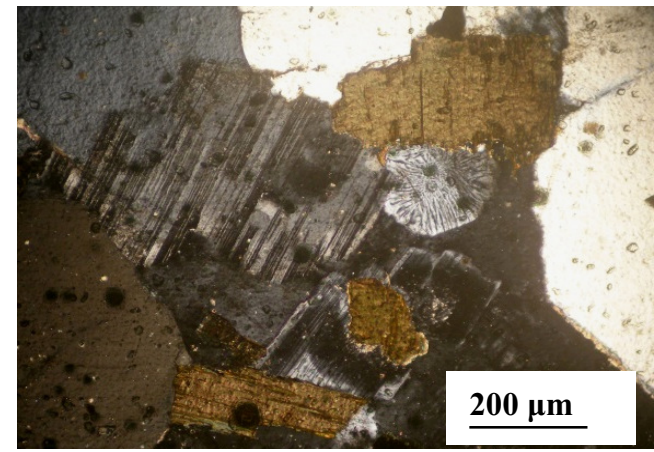

Fig.6:Photomicrograph for monzogranite of Wadi Murra area showing Oligoclase with myrmekitic textured and vermicular quartz, XPL

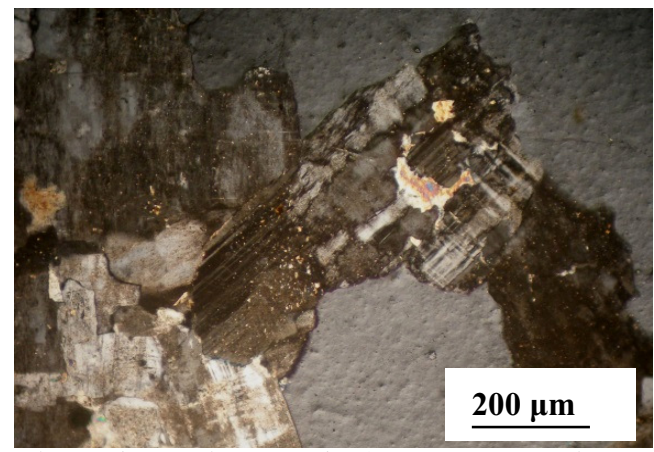

Fig.7:Photomicrograph for monzogranite of Wadi Murra area showing Coarse grained crystals of Qz enclosing finer crystals of albite, XPL.

ized by pleochroic haloes surrounding minute crystals of radioelements-bearing minerals (zircon), (Fig.8). Some flakes are partially chloritized excluding iron oxides as elongated blebs along cleavage planes (Fig.9) and others are transformed to penninite or muscovite (Fig.10). Muscovite is secondary mica mineral occurring after biotite (Figs.9\&10) or as irregular flakes secondarily-formed in late stages and corroding the earlier crystals of plagioclase (Fig.11).

Accessory minerals are represented mainly by allanite, epidote, zircon, fluoroapatite and 


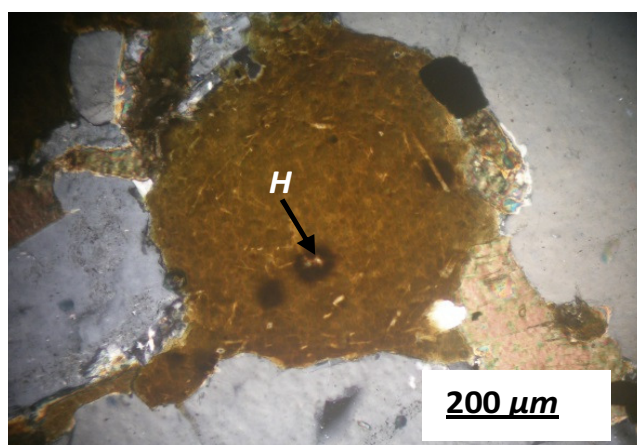

Fig.8:Photomicrograph for monzogranite of Wadi Murra area showing Pleochroic haloes of zircon in biotite, XPL

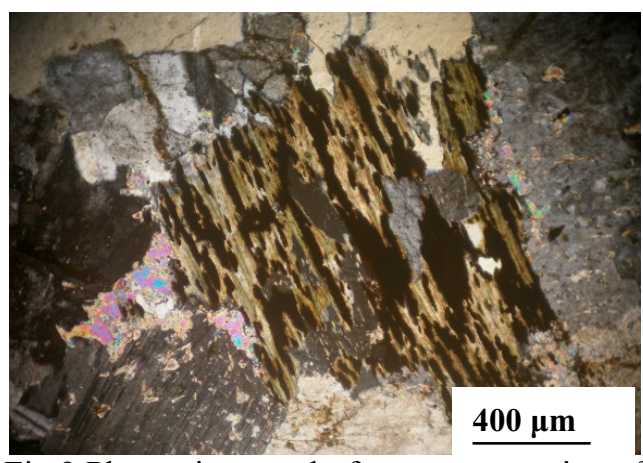

Fig.9:Photomicrograph for monzogranite of Wadi Murra area showing Biotite partially chloritized excluding iron oxides, XPL.

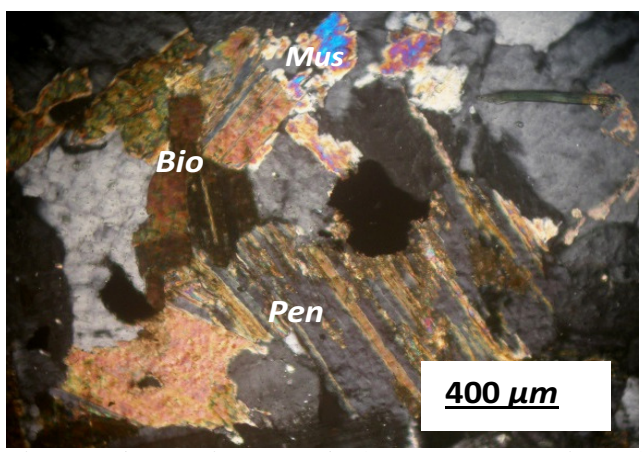

Fig.10:Photomicrograph for monzogranite of Wadi Murra area showing biotite partially transformed to chlorite, XPL

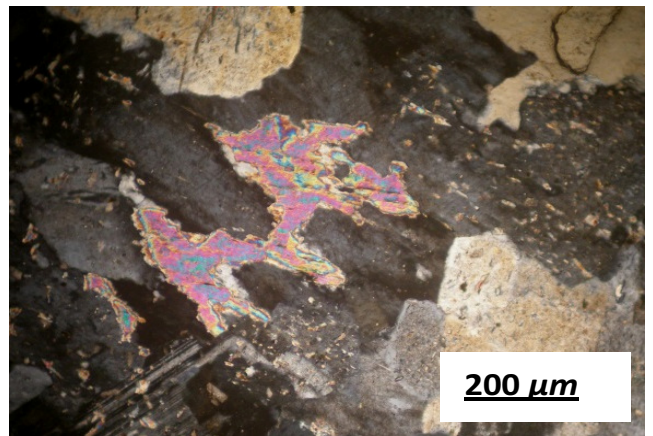

Fig.11:Photomicrograph for monzogranite of Wadi Murra area showing Muscovite as irregular flakes in association with biotite, XPL

sphene. Allanite is the most common accessory mineral displaying different varieties recognized by it scharacteristic yellowish brown colour. It occurs as well-formed crystals exhibiting its characteristic interference colours ( $3^{\text {rd }}$ order), (Fig.12) or as zoned crystals with variable interference colours related to the zonation (Fig.13). The crystals with masked interference colours are surrounded by radial fractures referring to the presence of radioelements in their crystal lattices. Epidote occurs as primary well-formed crystals associating plagioclase and exhibiting third order interference colours (Fig.14). Zircon occurs as wellformed crystal enclosed in biotite (Fig.15), some crystals are good carrier for the radioelements and characterized by pleochroic hallows in biotite. Fluoroapatite occurs as prismatic crystals oftenly enclosed in quartz and distinguished by its very low relief and characteristic interference colour (grey $2^{\text {nd }}$ order), (Fig.16). Sphene is present as well-formed primary crystals 4 with sphenoidal form (Fig.17) and as secondary anhedral crystals associating the chloritized biotite (Fig.18).

\section{Syenogranite}

The studied syenogranite is mediumgrained rock characterized by pink colour and equigranular texture composed of potash feldspars, plagioclase, quartz and biotite 


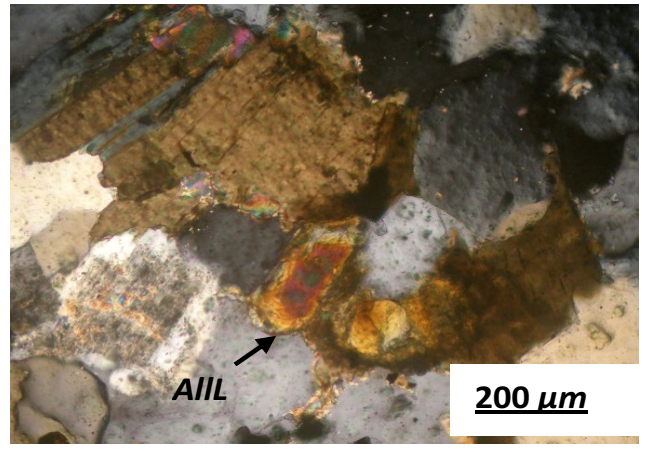

Fig.12:Photomicrograph for monzogranite of Wadi Murra area showing Allanite as wellformed crystals, XPL

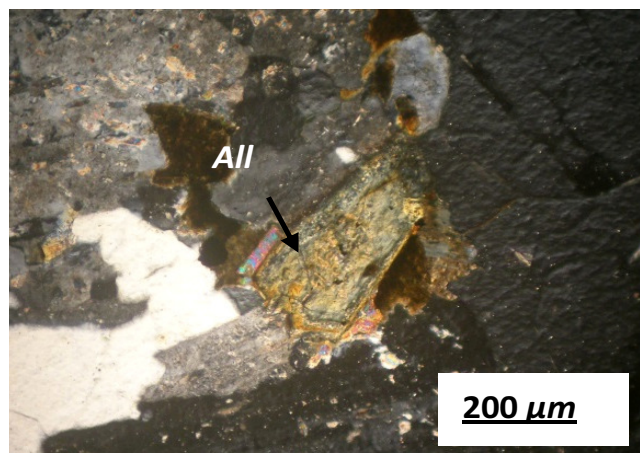

Fig.13:Photomicrograph for monzogranite of Wadi Murra area showing zoned crystals of allanite, XPL

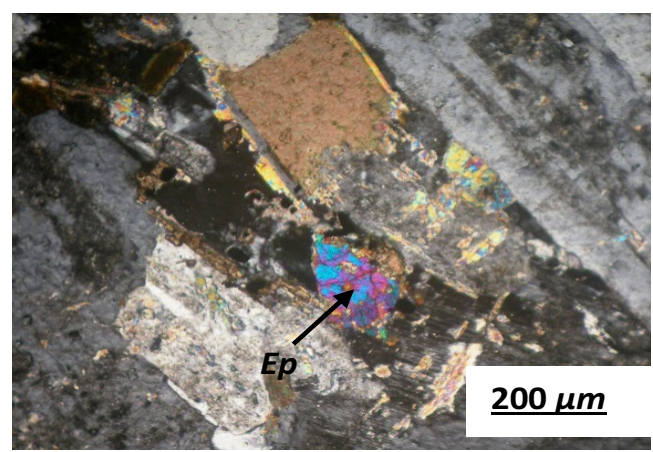

Fig.14:Photomicrograph for monzogranite of Wadi Murra area showing epidote as wellformed crystals associating plagioclase, XPL

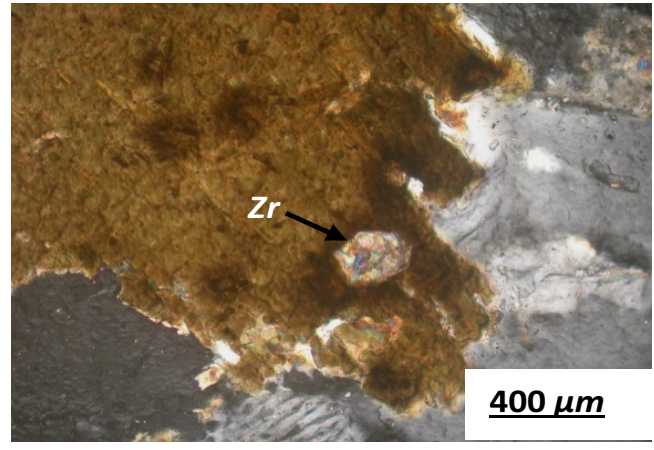

Fig.15:Photomicrograph for monzogranite of Wadi Murra area showing Zircon occurs as well-formed crystal enclosed in biotite, XPL

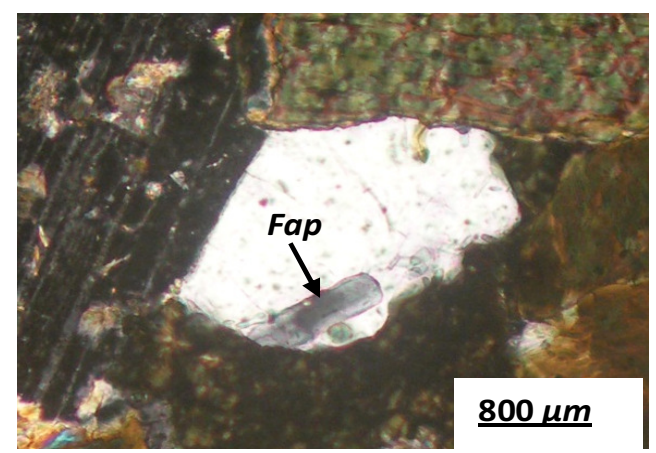

Fig.16:Photomicrograph for monzogranite of Wadi Murra area showing fluoroapatite as prismatic crystals oftenly enclosed in quartz,

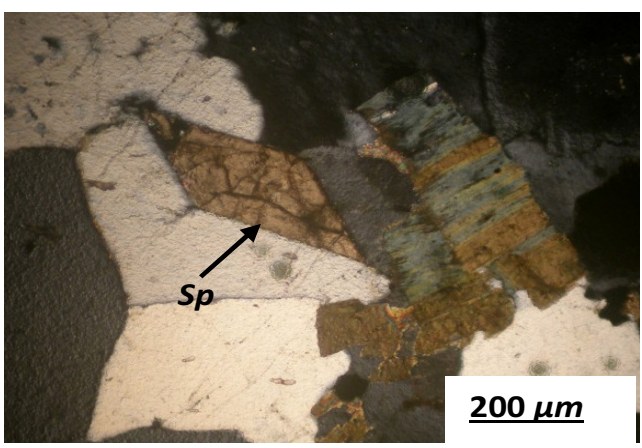

Fig.17:Photomicrograph for monzogranite of Wadi Murra area showing sphene as wellformed primary crystals with sphenoidal form, XPL 


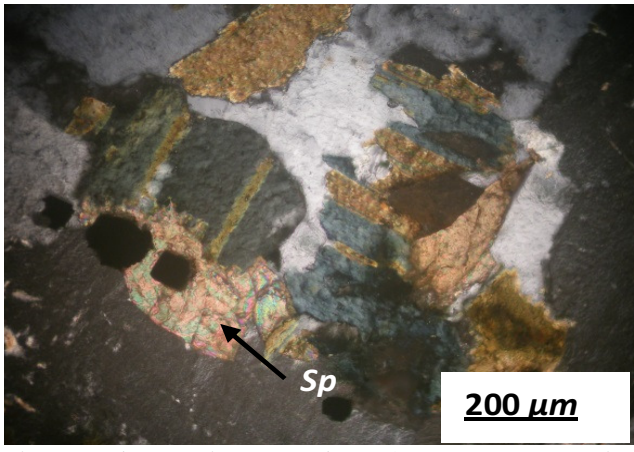

Fig.18:Photomicrograph for monzogranite of Wadi Murra area showing sphene as secondary anhedral crystals associating the chloritized biotite, XPL

as essential minerals and allanite, zircon and sphene as accessories.

Potash feldspars are more dominant than the plagioclase constitueting about $44.5 \%$ of the rock. They occur as anhedral crystals of microcline perthites and antiperthites, exhibiting its characteristic cross hatching twinning (Fig.19). The microcline perthite occurs as subhedral crystals of patchy perthite (Fig.20) and string perthite (Fig.21). Some crystals of perthite corrode the adjacent crystals of plagioclase referring to the sodic composition of plagioclase (Fig.22). Plagioclase $\left(\mathrm{An}_{12}\right)$ represents about $17.7 \%$ of the rock and occurs as subhedral crystals of oligoclase characterized by albitic twinning (Figs.20\&22). Quartz represents about $34.9 \%$ of the rock occurring as primary anhedral crystals associating the feldspars or as secondary fine crystals associating the metasomatized biotite (Fig.23).

Mafic minerals are represented only by biotite $(1.6 \%$ of the rock). It is present as intensively metasomatized flakes excluding iron oxides and secondary quartz along the cleavage planes (Fig.23).

Accessory minerals in Wadi Murra syenogranite are represented mainly by allanite, zircon, and sphene. Allanite is the most common accessory mineral displaying different

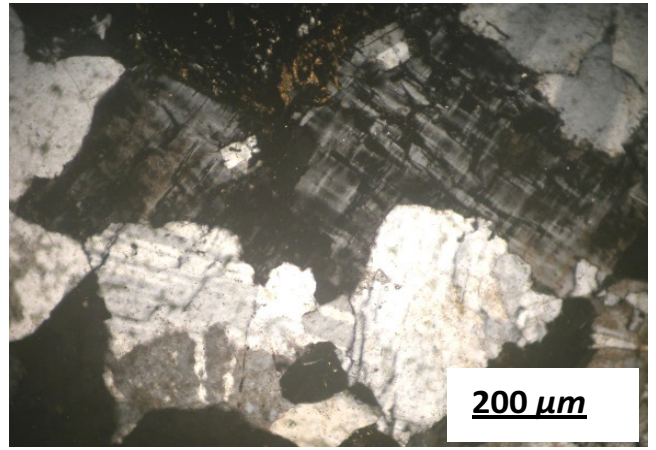

Fig.19:Photomicrograph for monzogranite of Wadi Murra area showing potash feldspars as anhedral crystals of microcline, XPL

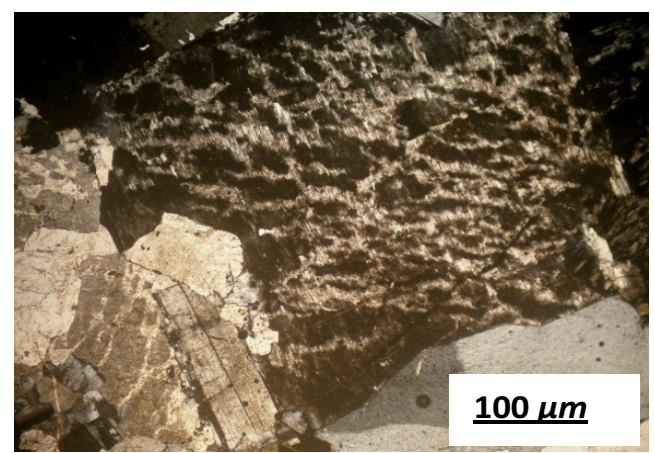

Fig.20:Photomicrograph for monzogranite of Wadi Murra area showing subhedral crystals of patchy perthite, XPL

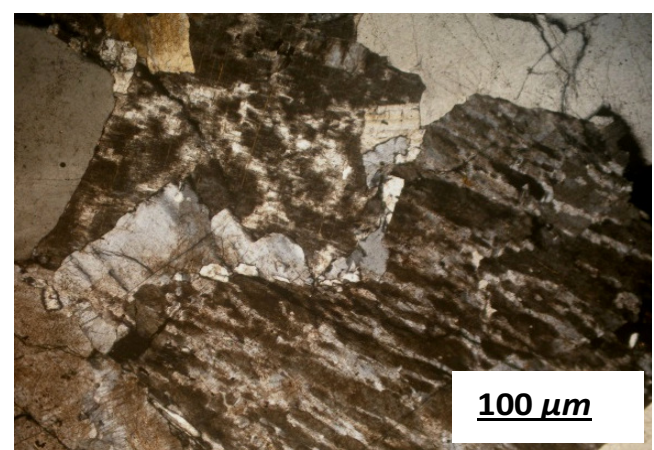

Fig.21:Photomicrograph for monzogranite of Wadi Murra area showing string perthite showing perthetic textures, XPL 


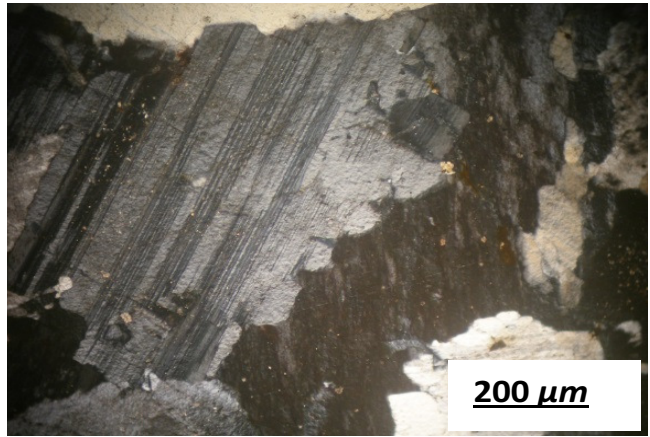

Fig.22:Photomicrograph for monzogranite of Wadi Murra area showing crystals of perthite corrode the adjacent crystals of plagioclase, XPL

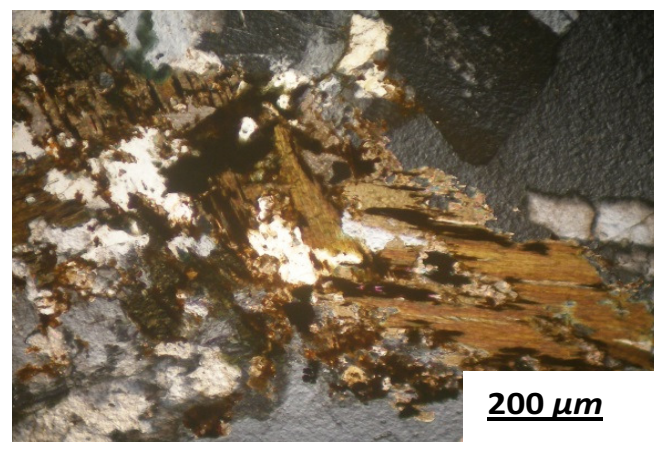

Fig.23:Photomicrograph for monzogranite of Wadi Murra area showing anhedral secondary fine crystals of qz associating the feldspars and metasomatized biotite, XPL

varieties and recognized by its characteristic yellowish brown colour. It occurs as wellformed crystals masked interference colours (Fig.24). Zircon is an accessory mineral occurring as well-formed crystals showing its characteristic interference colours of second order (Fig.25). Sphene is present as wellformed primary crystals with sphenoidal form associated biotite and plagioclase (Fig.26).

\section{Alkali Feldspar Granite}

Alkali feldspar granite is the most recent granite characterized by buff and pink co-

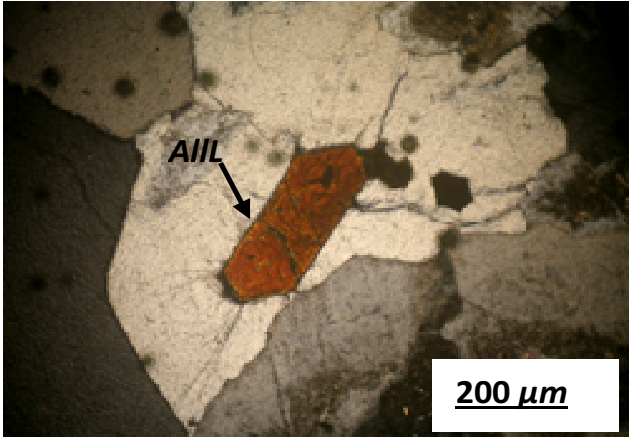

Fig.24:Photomicrograph for monzogranite of Wadi Murra area showing allanite with masked interference colours, XPL

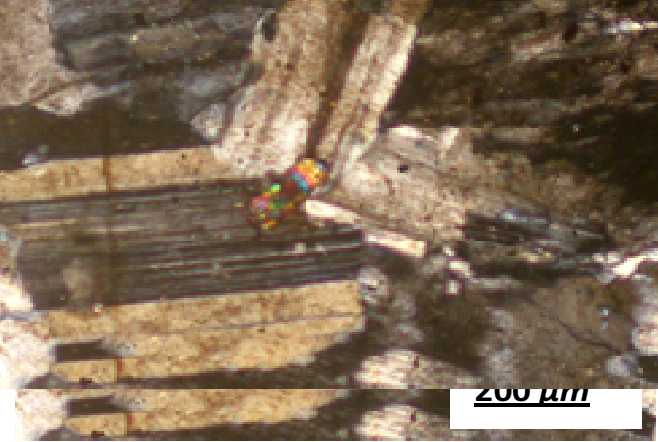

Fig.25:Photomicrograph for monzogranite of Wadi Murra area showing zircon as wellformed crystals associating zoned plagioclase, XPL

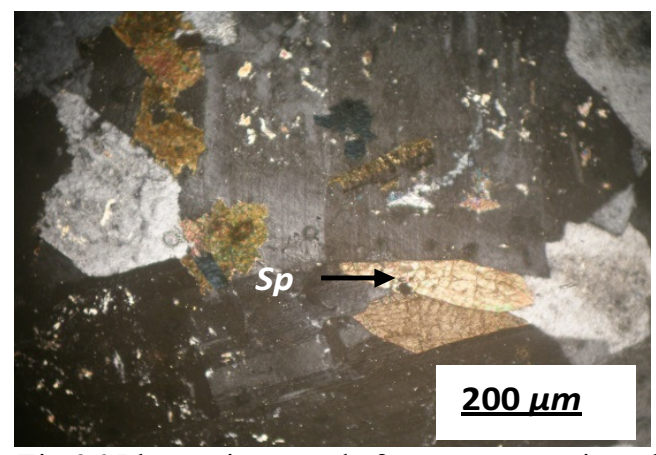

Fig.26:Photomicrograph for monzogranite of Wadi Murra area showing sphene as wellformed primary crystals with sphenoidal form, XPL 
lours due to the dominance of the alkali-feldsaprs. It is medium-grained rock characterized by equigranular texture and consists of potash feldspar, plagioclase, quartz, and biotite as essential minerals and allanite, zircon and sphene as accessories.

Potash feldspar is the main feldspar occupying $60.6 \%$ of the rock. It occurs mostly as euhedral crystals of microcline exhibiting its characteristic cross-hatched twinning (Fig.27). Dominance of microcline refers to low temperature of crystallization and shallow depth for formation. The potash feldspars is also present as perthite of feather type and microcline perthite (Fig.28). Antiperthite crystals are also present as euhedral crystals of patchy type (Fig.29). Plagioclase $\left(\mathrm{An}_{6}\right)$ represents about $2.8 \%$ of the rock. It is present as subhedral fine crystals of albite interstitial between quartz and perthite exhibiting its characteristic albitic twinning (Fig.30). Quartz occupies about $34.8 \%$ of the rock and occurs as anhedral crystal associating the other constituents and exhibits wavy extinction (Fig.31).

Mafic minerals represent about $0.9 \%$ of the rock. It occurs as flakes of biotite enclosing the opaque minerals as well-formed primary crystals or as secondary anhedral minute crystals distributed along their cleavage planes (Figs.31\&32). Biotite flakes also enclose the zircon crystals as accessory mineral that present as well-formed crystals showing normal interference colours (Fig.32).

Accessory minerals in Wadi Murra alkali feldspar granite are represent by allanite, zircon, and sphene. Allanite is the most common accessory minerals displaying different varieties recognized by its characteristic yellowish brown colour. It occurs as wellformed crystals masked interference colours (Fig.33) or as zoned crystals with variable interference colours related to the zonation associated biotite, quartz and sphene (Fig.34). Zircon is an accessory mineral occurring as well-formed crystals showing its characteristic interference colours of second order enclosed in plagioclase (Fig.36). Sphene is present as

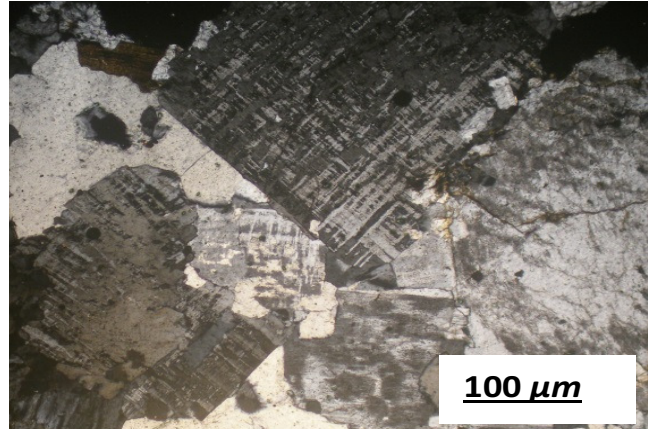

Fig.27: Photomicrograph for monzogranite of Wadi Murra area showing crystals of microcline with characteristic cross-hatched twining, XPL

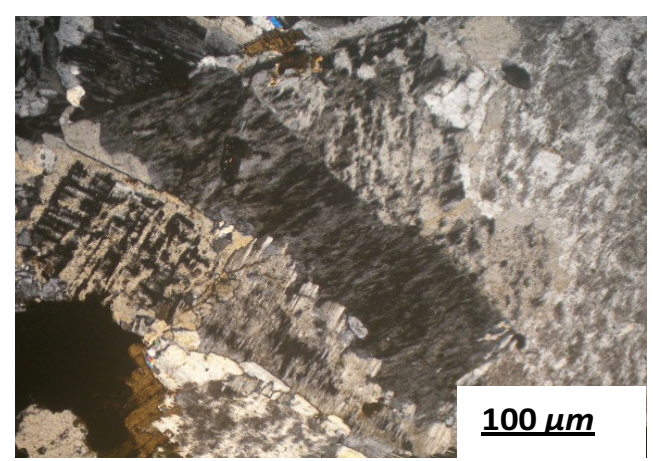

Fig.28:Photomicrograph for monzogranite of Wadi Murra area showing perthite of feather type and microcline perthite, XPL

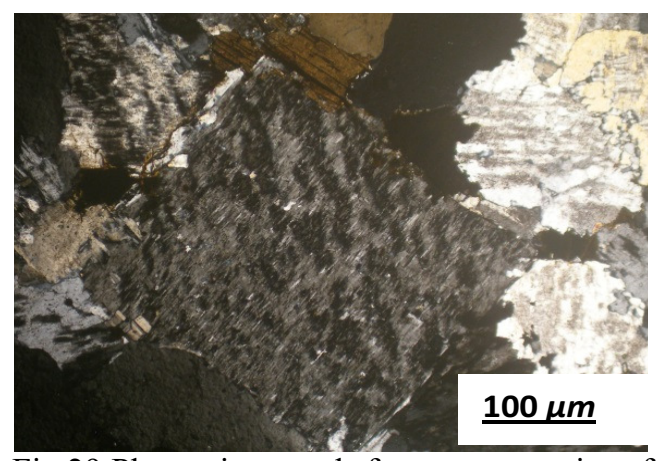

Fig.29:Photomicrograph for monzogranite of Wadi Murra area showing euhedral crystals of patchy antiperthite, XPL 


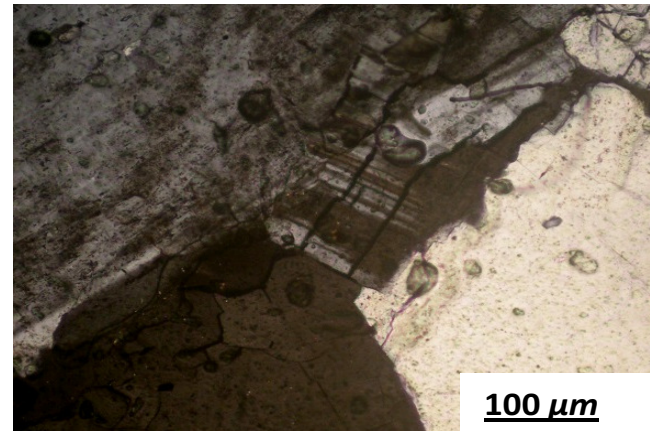

Fig.30:Photomicrograph for monzogranite of Wadi Murra area showing subhedral fine crystals of albite interstitial between quartz and perthite, XPL

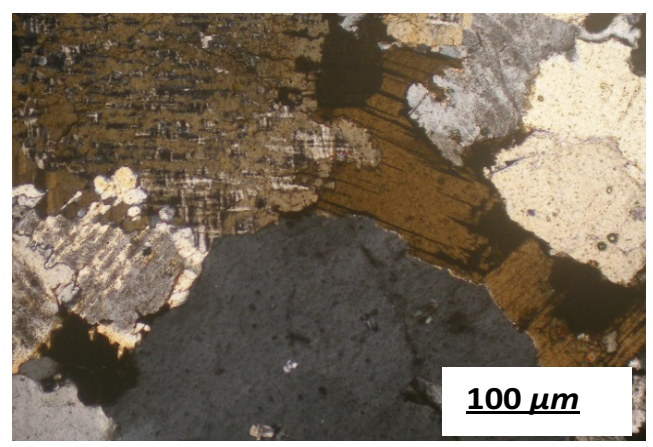

Fig.31:Photomicrograph for monzogranite of Wadi Murra area showing anhedral crystal of quartz showing wavy extinction, XPL

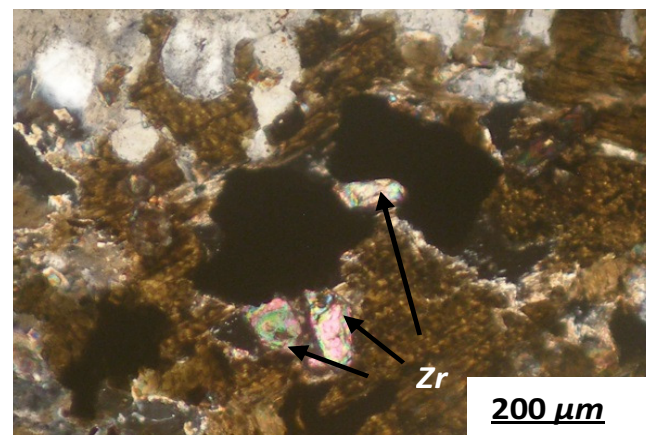

Fig.32:Photomicrograph for monzogranite of Wadi Murra area showing biotite flakes encloses zircon crystals associated opaque minerals, XPL

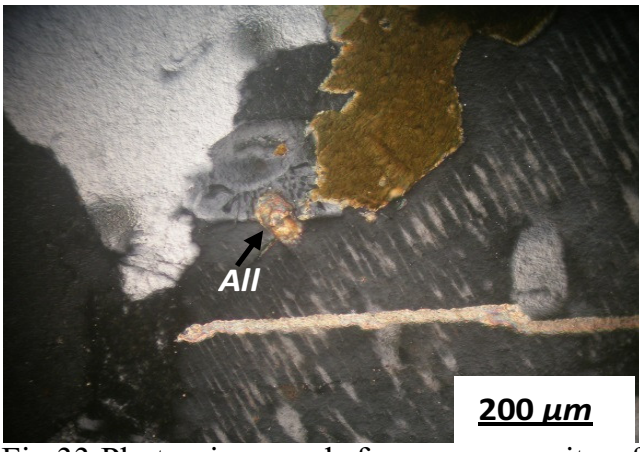

Fig.33:Photomicrograph for monzogranite of Wadi Murra area showing allanite as wellformed crystals associated biotite, quartz and string perthite, XPL

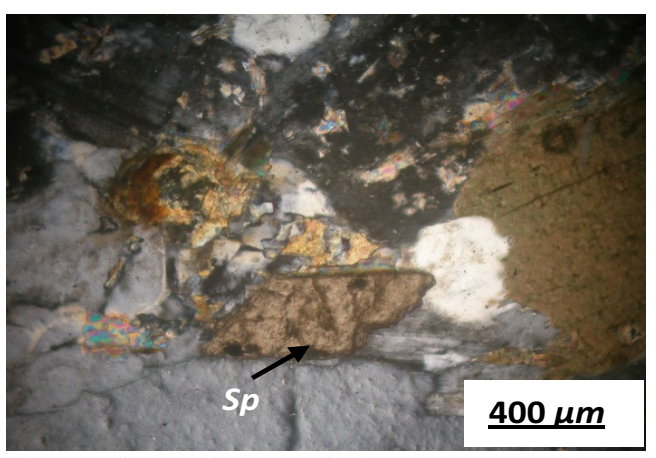

Fig.34:Photomicrograph for monzogranite of Wadi Murra area showing zoned allanite as well-formed crystals associated biotite, quartz and sphene, XPL

well-formed primary crystals with sphenoidal form associated biotite and quartz (Fig.34) and present as secondary anhedral crystals associating the choloritized biotite, quartz and plagioclase (Fig.35).

\section{GEOCHEMISTRY}

The geochemical characteristics of Wadi Murra granites were investigated through the chemical analyses of twenty two samples (9 monzogranites, 5 syenogranites and 6 alkali feldspar granites) were selected for major 


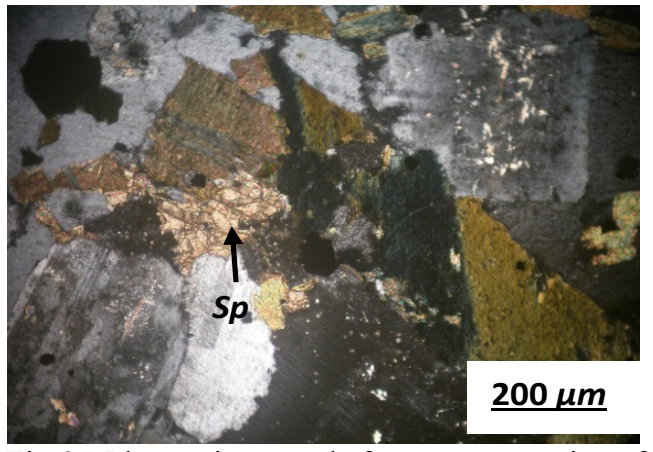

Fig.35:Photomicrograph for monzogranite of Wadi Murra area showing sphene as secondary anhedral crystals associating the chloritized biotite, XPL

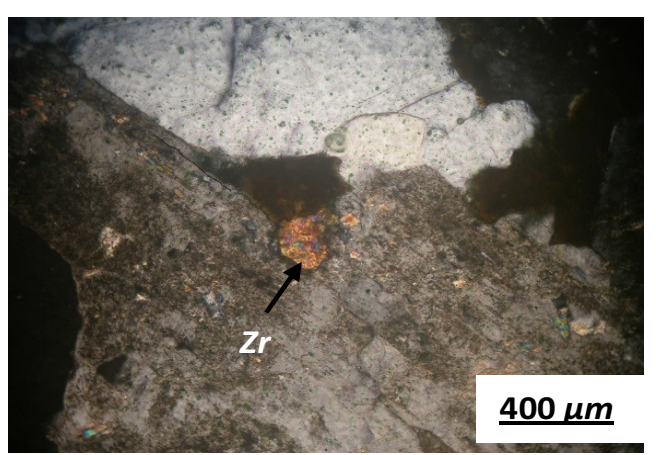

Fig.36:Photomicrograph for monzogranite of Wadi Murra area showing zircon as wellformed crystals enclosed in plagioclase, XPL

oxides, some trace elements and rare earth elements analyses. The analyses of the major oxides and some trace elements were completed at Laboratories of Nuclear Materials Authority (NMA), Egypt. The rare earth elements were determined by inductively coupled plasma-mass spectrometry (ICP-MS) at the ACME Analytical Laboratories Ltd., Canada. The results of the chemical analyses are shown in Table (1).

Representative major oxides and trace elements analyses for the different granitic rocks types from Wadi Murra area have been classi- fied using the R1-R2 diagram of De La Roche et al.(1980), (Fig.37) and the ratio between P and Q of Debon and Fort (1983), (Fig.38). The studied younger granite samples plot in the monzogranite, syenogranite and alkali feldspar fields.

The variation of $\left(\mathrm{Na}_{2} \mathrm{O}+\mathrm{K}_{2} \mathrm{O}\right)$ versus $\mathrm{SiO}_{2}$ has been considered as an effective means for distinguishing alkaline from subalkaline rocks. Irvine and Baragar (1971) diagram (Fig.39) shows that the studied granites fall in the subalkaline field suggesting closer association with orogenic tectonic environments.

Alumina saturation diagram $\left(\mathrm{Al}_{2} \mathrm{O}_{3}-\mathrm{CaO}-\right.$ $\left(\mathrm{Na}_{2} \mathrm{O}+\mathrm{K}_{2} \mathrm{O}\right)$ of Shand (1951) is used to distinguish between peraluminous, metaluminous and peralkaline granitic rock types (Fig.40). The figure shows that the studied monzogranite and syenogranite are located on the border between the metaluminous and peraluminous over the line of unity indicating that they are mostly metaluminous (appreciable alumina saturation). On the other hand, alkali-feldspar granite is completely falling in the field of peralkaline field.

\section{Tectonic Setting}

Tectonic setting of the post collision granites of Wadi Murra granites are concluded by plotting the analyzed samples on the $\mathrm{Al}_{2} \mathrm{O}_{3}$ $\mathrm{SiO}_{2}$ binary discrimination diagram of Maniar and Piccoli (1989). The analyzed samples plot in the post-orogenic granite (POG) field, (Fig.41). Plotting on the $\mathrm{Rb}$ vs $(\mathrm{Y}+\mathrm{Nb})$ discrimination diagram of Pearce et al. (1984) showing that the studied granites plotted in the within-plate granite (WPG) except two samples of monzogranites and two samples of syenogranites plotted in the volcanic arc granite field (Fig.42). All the younger granite samples plot in Post Collision granite field of Pearce (1996).

On the $\mathrm{FeO}_{t} / \mathrm{MgO}$ and $\left(\mathrm{K}_{2} \mathrm{O}+\mathrm{Na}_{2} \mathrm{O}\right) / \mathrm{CaO}$ versus $(\mathrm{Zr}+\mathrm{Nb}+\mathrm{Ce}+\mathrm{Y})$ diagrams after Whalen et al. (1987), the studied granites plotted in the A-type granite field, (Figs. 43\&44), and plot 
Table 1:Major oxides, some trace elements and rare earth elements for the studied younger granites in Wadi Murra area, South Eastern Desert, Egypt

\begin{tabular}{|c|c|c|c|c|c|c|c|c|c|c|c|c|c|c|}
\hline & 1 & 2 & 3 & 4 & 5 & 6 & 7 & 8 & 9 & Av. & 10 & 11 & 12 & 13 \\
\hline $\mathrm{SiO}_{2}$ & 71.62 & 71.41 & 72.78 & 72.09 & 71.73 & 73.05 & 72.88 & 73.04 & 72.51 & 72.34 & 73.55 & 73.3 & 73.42 & 73.33 \\
\hline $\mathrm{TiO}_{2}$ & 0.2 & 0.23 & 0.15 & 0.13 & 0.27 & 0.06 & 0.04 & 0.12 & 0.19 & 0.15 & 0.19 & 0.11 & 0.2 & 0.21 \\
\hline $\mathrm{Al}_{2} \mathrm{O}_{3}$ & 14.04 & 13.55 & 13.37 & 13.34 & 13.61 & 13.65 & 13.81 & 14.19 & 14.02 & 13.73 & 13.07 & 13.11 & 12.58 & 12.49 \\
\hline $\mathrm{Fe}_{2} \mathrm{O}_{3}$ & 1.76 & 1.74 & 1.97 & 1.62 & 1.85 & 1.5 & 1.38 & 1.54 & 1.24 & 1.62 & 1.95 & 1.96 & 1.75 & 1.76 \\
\hline $\mathrm{FeO}$ & 1.48 & 1.47 & 1.77 & 0.91 & 1.56 & 1.02 & 0.79 & 1.38 & 0.97 & 1.26 & 1.81 & 1.82 & 1.53 & 1.52 \\
\hline $\mathrm{MnO}$ & 0.06 & 0.06 & 0.05 & 0.06 & 0.06 & 0.01 & 0.03 & 0.02 & 0.05 & 0.04 & 0.02 & 0.03 & 0.04 & 0.03 \\
\hline MgO & 0.65 & 0.64 & 0.11 & 0.59 & 0.81 & 0.07 & 0.03 & 0.03 & 0.66 & 0.39 & 0.08 & 0.1 & 0.14 & 0.13 \\
\hline $\mathrm{CaO}$ & 1.31 & 1.29 & 1.25 & 1.6 & 1.55 & 1.61 & 1.41 & 0.9 & 1.36 & 1.36 & 0.65 & 0.76 & 1 & 1.01 \\
\hline $\mathrm{Na}_{2} \mathrm{O}$ & 4.2 & 4.22 & 4.12 & 4.4 & 4.27 & 4.55 & 4.81 & 4.49 & 4.12 & 4.35 & 4.33 & 4.37 & 4.64 & 4.7 \\
\hline $\mathrm{K}_{2} \mathrm{O}$ & 3.67 & 3.75 & 3.78 & 4.06 & 3.66 & 3.62 & 4.07 & 3.59 & 3.95 & 3.79 & 3.83 & 3.82 & 4.13 & 4.15 \\
\hline $\mathrm{P}_{2} \mathrm{O}_{5}$ & 0.12 & 0.14 & 0.02 & 0.09 & 0.15 & 0.01 & 0.01 & 0.01 & 0.13 & 0.075 & 0.01 & 0.02 & 0.02 & 0.01 \\
\hline LOI & 0.42 & 0.6 & 0.73 & 0.85 & 0.5 & 0.84 & 0.58 & 0.76 & 0.44 & 0.63 & 0.65 & 0.66 & 0.44 & 0.43 \\
\hline \multicolumn{15}{|c|}{ Some Norm Values } \\
\hline Qz & 27.05 & 28.83 & 30.87 & 26.85 & 28.09 & 29.05 & 26.3 & 30.75 & 29.7 & 28.61 & 31.45 & 30.86 & 28.52 & 28.15 \\
\hline Or & 23.59 & 22.52 & 22.5 & 24.28 & 21.75 & 21.6 & 24.25 & 21.4 & 23.57 & 22.82 & 22.83 & 22.73 & 24.56 & 24.27 \\
\hline $\mathbf{A b}$ & 35.74 & 36.21 & 35.01 & 37.6 & 36.26 & 38.78 & 40.96 & 38.25 & 35.12 & 37.10 & 36.87 & 37.16 & 39.43 & 30.09 \\
\hline An & 5.84 & 5.67 & 6.13 & 4.67 & 6.85 & 6.15 & 4.06 & 4.44 & 5.74 & 5.50 & 5.19 & 3.68 & 1.37 & 0.7 \\
\hline \multicolumn{15}{|c|}{ Treace Elements } \\
\hline $\mathbf{B a}$ & 25 & 559 & 117 & 352 & 517 & 242 & 16 & 15 & 461 & 256 & 97 & 98 & 83 & 85 \\
\hline $\mathbf{R b}$ & 61 & 99 & 60 & 134 & 126 & 101 & 154 & 176 & 116 & 114.1 & 42 & 49 & 54 & 55 \\
\hline $\mathrm{Sr}$ & 15 & 187 & 45 & 131 & 214 & 87 & 8 & 8 & 184 & 97.6 & 36 & 39 & 31 & 32 \\
\hline Ga & 10 & 20 & 23 & 27 & 25 & 27 & 36 & 41 & 23 & 25.77 & 24 & 25 & 25 & 26 \\
\hline $\mathbf{Y}$ & 271 & 34 & 21 & 79 & 54 & 18 & 50 & 104 & 59 & 76.67 & 25 & 27 & 97 & 70 \\
\hline $\mathrm{Zr}$ & 591 & 135 & 174 & 281 & 177 & 147 & 128 & 136 & 173 & 215.7 & 134 & 140 & 150 & 152 \\
\hline $\mathrm{Nb}$ & 101 & 22 & 23 & 33 & 21 & 21 & 58 & 30 & 21 & 36.66 & 12 & 15 & 19 & 19 \\
\hline Th & 8.53 & 11.9 & 8.5 & 23.9 & 13.7 & 9.4 & 12.6 & 16.7 & 19 & 13.80 & 8.6 & 8.6 & 7.4 & 7.4 \\
\hline $\mathbf{U}$ & 2.52 & 4 & 2.5 & 8 & 4.5 & 3.2 & 6.2 & 5.6 & 4.2 & 4.524 & 2.5 & 2.5 & 2.4 & 2.4 \\
\hline $\mathrm{Cr}$ & 27 & 10 & 11 & 14 & 22 & 15 & 13 & 9 & 20 & 15.66 & 12 & 16 & 10 & 11 \\
\hline $\mathbf{N i}$ & 7 & 2 & 1 & 4 & 4 & 1 & 1 & 1 & 3 & 2.66 & 1 & 1 & 1 & 1 \\
\hline V & 5 & 24 & 5 & 21 & 34 & 5 & 1 & 1 & 25 & 13.4 & 3 & 4 & 5 & 6 \\
\hline $\mathrm{Cu}$ & 9 & 10 & 5 & 23 & 20 & 6 & 29 & 12 & 17 & 14.5 & 27 & 27 & 5 & 5 \\
\hline $\mathrm{Pb}$ & 20 & 25 & 18 & 36 & 25 & 33 & 38 & 42 & 25 & 29.11 & 17 & 16 & 18 & 18 \\
\hline Zn & 108 & 81 & 97 & 176 & 121 & 57 & 137 & 298 & 91 & 129.5 & 107 & 101 & 136 & 136 \\
\hline \multicolumn{15}{|c|}{ Rare Earth Elements } \\
\hline La & 26.55 & 87.6 & 36 & 60.8 & 41.5 & 44 & 55 & 25.2 & 40.4 & 46.33 & 66.1 & 70 & 76.7 & 80 \\
\hline $\mathrm{Ce}$ & 63.58 & 196.4 & 82.82 & 140.7 & 94.99 & 57.11 & 96.12 & 61.46 & 91.64 & 98.31 & 151.5 & 155 & 184.1 & 180 \\
\hline $\operatorname{Pr}$ & 9.25 & 26.3 & 10.8 & 19 & 12.5 & 18 & 24 & 9.8 & 12.4 & 15.78 & 20.2 & 25.3 & 26 & 27.2 \\
\hline Nd & 47.42 & 89.9 & 39.1 & 65.3 & 43.8 & 52 & 47 & 21.3 & 45 & 50.09 & 72.1 & 70.3 & 90.9 & 90.1 \\
\hline Sm & 12.92 & 17.9 & 8.8 & 14.9 & 10.9 & 21 & 23 & 6.1 & 9.9 & 13.93 & 13.9 & 14.1 & 21.5 & 20.5 \\
\hline Eu & 0.91 & 0.8 & 1.2 & 1 & 1 & 0.8 & 0.9 & 0.9 & 1.2 & 0.967 & 0.7 & 0.72 & 0.9 & 0.91 \\
\hline Gd & 11.62 & 11 & 6.8 & 13.2 & 9.6 & 12.2 & 11.9 & 6.8 & 8.8 & 10.21 & 9.1 & 10.2 & 17.6 & 16.2 \\
\hline $\mathbf{T b}$ & 2.31 & 1.4 & 1.3 & 2.6 & 1.8 & 1.4 & 1.7 & 1.6 & 1.8 & 1.767 & 1.3 & 1.26 & 3.3 & 4.1 \\
\hline Dy & 13.5 & 16 & 9.8 & 14.5 & 6.3 & 12.7 & 14.9 & 10.9 & 9.9 & 12.05 & 6.7 & 7.5 & 16 & 17.3 \\
\hline Ho & 2.79 & 1.2 & 1.5 & 3.2 & 2.1 & 1.9 & 2.4 & 2.5 & 2.2 & 2.19 & 1.5 & 1.6 & 3.3 & 3.92 \\
\hline $\mathbf{E r}$ & 9.04 & 2.5 & 3.7 & 8.3 & 5.9 & 3.1 & 3.8 & 6.9 & 5.8 & 5.44 & 4.8 & 5.76 & 7.6 & 8.12 \\
\hline $\mathrm{Tm}$ & 0.67 & 0.4 & 0.6 & 1.5 & 0.9 & 1.2 & 1.3 & 1.1 & 1 & 0.96 & 0.65 & 0.75 & 1.2 & 1.22 \\
\hline $\mathbf{Y b}$ & 3.52 & 2.6 & 3.5 & 8.5 & 5.3 & 5.7 & 6.6 & 5.6 & 5.9 & 5.24 & 3.5 & 3.28 & 6.3 & 7.33 \\
\hline Lu & 0.35 & 0.4 & 0.6 & 1.3 & 0.9 & 0.7 & 0.8 & 0.9 & 0.8 & 0.75 & 0.5 & 0.51 & 1 & 1.1 \\
\hline TREE & 204.43 & 454.4 & 206.52 & 354.8 & 237.49 & 231.81 & 289.42 & 161.06 & 236.74 & 264.07 & 352.55 & 366.28 & 456.4 & 458 \\
\hline LREE & 159.72 & 418.1 & 177.52 & 300.7 & 203.69 & 192.11 & 245.12 & 123.86 & 199.34 & 224.46 & 323.8 & 334.7 & 399.2 & 397.8 \\
\hline HREE & 44.71 & 36.3 & 29 & 54.1 & 33.8 & 39.7 & 44.3 & 37.2 & 37.4 & 39.612 & 28.75 & 31.58 & 57.2 & 60.2 \\
\hline
\end{tabular}


Table 1: Continued

\begin{tabular}{|c|c|c|c|c|c|c|c|c|c|}
\hline & 14 & Av. & 15 & 16 & 17 & 18 & 19 & 20 & Av. \\
\hline $\mathrm{SiO}_{2}$ & 73.57 & 73.434 & 74.62 & 74.38 & 74.31 & 74.6 & 75.45 & 74.06 & 74.57 \\
\hline $\mathrm{TiO}_{2}$ & 0.14 & 0.17 & 0.11 & 0.32 & 0.32 & 0.41 & 0.4 & 0.3 & 0.31 \\
\hline $\mathbf{A l}_{2} \mathbf{O}_{3}$ & 12.72 & 12.794 & 12.27 & 12.16 & 12.11 & 11.67 & 11.26 & 12.26 & 11.95 \\
\hline $\mathrm{Fe}_{2} \mathrm{O}_{3}$ & 1.84 & 1.852 & 1.42 & 1.57 & 1.76 & 1.76 & 1.85 & 1.87 & 1.705 \\
\hline FeO & 1.65 & 1.666 & 1.27 & 1.38 & 1.19 & 1.36 & 1.27 & 1.08 & 1.258 \\
\hline MnO & 0.05 & 0.034 & 0.04 & 0.03 & 0.03 & 0.06 & 0.05 & 0.05 & 0.043 \\
\hline MgO & 0.09 & 0.108 & 0.13 & 0.11 & 0.31 & 0.43 & 0.62 & 0.26 & 0.31 \\
\hline $\mathrm{CaO}$ & 0.73 & 0.83 & 0.54 & 0.64 & 0.56 & 0.63 & 0.34 & 0.57 & 0.546 \\
\hline $\mathrm{Na}_{2} \mathbf{O}$ & 4.5 & 4.508 & 4.5 & 4.6 & 4.56 & 4.02 & 4.01 & 4.64 & 4.388 \\
\hline $\mathbf{K}_{2} \mathbf{O}$ & 3.91 & 3.968 & 4.82 & 4.84 & 4.34 & 4.33 & 4.32 & 4.25 & 4.483 \\
\hline $\mathbf{P}_{2} \mathbf{O}_{5}$ & 0.02 & 0.016 & 0.02 & 0.01 & 0.06 & 0.14 & 0.13 & 0.07 & 0.071 \\
\hline LOI & 0.61 & 0.558 & 0.33 & 0.34 & 0.65 & 0.31 & o.3 & 0.58 & 0.418 \\
\hline \multicolumn{10}{|c|}{ Some Norm Values } \\
\hline $\mathbf{Q z}$ & 30.33 & 29.862 & 28.91 & 28 & 29.61 & 32.82 & 33.8 & 29.18 & 30.38 \\
\hline Or & 23.31 & 23.54 & 28.58 & 28.67 & 25.79 & 25.76 & 25.63 & 25.3 & 26.62 \\
\hline Ab & 38.33 & 36.376 & 36.26 & 35.55 & 38.19 & 34.18 & 33.87 & 39.24 & 36.21 \\
\hline An & 2.95 & 2.778 & 0.2 & 0.1 & o.1 & 0.98 & 0.12 & 0.15 & 0.275 \\
\hline \multicolumn{10}{|c|}{ Trace Elements } \\
\hline Ba & 106 & 93.8 & 31 & 74 & 274 & 51 & 468 & 276 & 195.67 \\
\hline $\mathbf{R b}$ & 52 & 50.4 & 85 & 182 & 100 & 136 & 131 & 188 & 137 \\
\hline $\mathbf{S r}$ & 32 & 34 & 15 & 30 & 95 & 29 & 154 & 96 & 69.83 \\
\hline $\mathbf{G a}$ & 23 & 24.6 & 30 & 37 & 25 & 26 & 25 & 28 & 28.5 \\
\hline $\mathbf{Y}$ & 39 & 51.6 & 387 & 152 & 101 & 353 & 75 & 113 & 196.83 \\
\hline $\mathbf{Z r}$ & 87 & 132.6 & 859 & 361 & 230 & 787 & 201 & 223 & 443.5 \\
\hline $\mathbf{N b}$ & 16 & 16.2 & 101 & 41 & 32 & 133 & 38 & 34 & 63.16 \\
\hline Th & 10.3 & 8.46 & 16.4 & 16.9 & 24.4 & 27.1 & 27.1 & 15 & 21.15 \\
\hline $\mathbf{U}$ & 1.9 & 2.34 & 5.2 & 5.4 & 4.8 & 9.8 & 9.8 & 4.9 & 6.65 \\
\hline $\mathbf{C r}$ & 16 & 13 & 30 & 21 & 11 & 29 & 17 & 10 & 19.66 \\
\hline $\mathbf{N i}$ & 1 & $\mathbf{1}$ & 7 & 2 & 2 & 7 & 3 & 11 & 5.33 \\
\hline $\mathbf{V}$ & 4 & 4.4 & 5 & 5 & 15 & 11 & 27 & 17 & 13.33 \\
\hline $\mathbf{C u}$ & 9 & 14.6 & 10 & 9 & 13 & 12 & 24 & 13 & 13.5 \\
\hline $\mathbf{P b}$ & 18 & 17.4 & 20 & 44 & 31 & 36 & 39 & 12 & 30.33 \\
\hline Zn. & 91. & 114.2 & 158 & 259 & 170 & 198 & 117 & 179 & 180.17. \\
\hline \multicolumn{10}{|c|}{ Rare Earth Elements } \\
\hline La & 98.9 & 78.34 & 38.24 & 37.8 & 60 & 32.88 & 50.6 & 66.1 & 47.603 \\
\hline Ce & 207.3 & $\mathbf{1 7 5 . 5 8}$ & 106.8 & 95.02 & 200 & 92.29 & 113.9 & 153.9 & 126.99 \\
\hline Pr & 28.4 & 25.42 & 15.24 & 13.1 & 18 & 13.76 & 15.2 & 21.6 & 16.15 \\
\hline Nd & 100.4 & 84.76 & 73.54 & 46.2 & 70.1 & 26.39 & 51.9 & 76.5 & $\mathbf{5 7 . 4 3}$ \\
\hline Sm & 19.8 & 17.96 & 18.61 & 13.8 & 20.5 & 9.52 & 13 & 19.6 & 15.83 \\
\hline Eu & 0.8 & 0.806 & 0.85 & 0.3 & 0.55 & 0.51 & 0.8 & 0.9 & 0.651 \\
\hline Gd & 13.8 & 13.38 & 12.73 & 14.3 & 18.7 & 15.98 & 12.5 & 21.4 & 15.93 \\
\hline Tb & 2 & 2.392 & 2.46 & 3.2 & 4.5 & 2.23 & 2.1 & 3.8 & 3.048 \\
\hline Dy & 18.3 & 13.16 & 17.2 & 17.2 & 20.3 & 16.06 & 11.9 & 20.7 & 17.22 \\
\hline Ho & 2.8 & 2.624 & 2.65 & 4 & 5.2 & 2.2 & 2.7 & 4.6 & 3.558 \\
\hline Er & 9 & 7.056 & 4.78 & 10 & 12.8 & 12.42 & 7.7 & 11.5 & 9.866 \\
\hline $\mathbf{T m}$ & 0.9 & 0.944 & 1.48 & 1.6 & 2.3 & 1.1 & 1.4 & 1.8 & 1.613 \\
\hline $\mathbf{Y b}$ & 6.1 & 5.302 & 7.38 & 9.5 & 11 & 7.92 & 8.7 & 10.2 & 9.116 \\
\hline Lu & 0.5 & 0.722 & 2.64 & 1.5 & 1.25 & 1.2 & 1.3 & 1.5 & 1.56 \\
\hline TREE & 509 & 428.446 & 304.6 & 267.52 & 445.2 & 234.46 & 293.7 & 414.1 & 326.6 \\
\hline LREE & 454.8 & 382.06 & 252.43 & 205.92 & 368.6 & 174.84 & 244.6 & 337.7 & 264.02 \\
\hline HREE & 54.2 & 46.386 & 52.17 & 61.6 & 76.6 & 59.62 & 49.1 & 76.4 & 62.58 \\
\hline
\end{tabular}




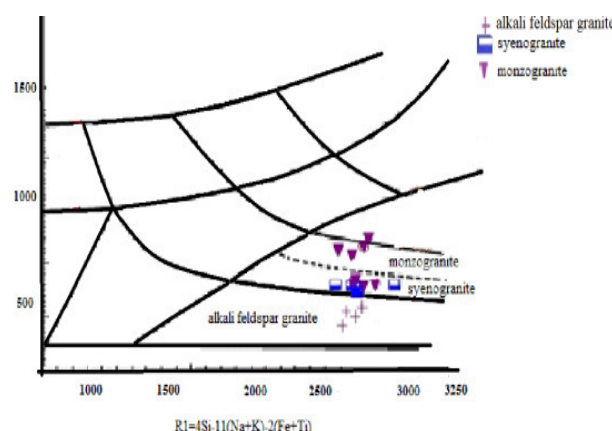

Fig.37:R1-R2 binary diagram (De La Roche et al.1980), $\mathrm{R} 1=4 \mathrm{Si}-11(\mathrm{Na}+\mathrm{K})-2(\mathrm{Fe}+\mathrm{Ti})$, $\mathrm{R} 2=6 \mathrm{Ca}+2 \mathrm{Mg}+\mathrm{Al}$

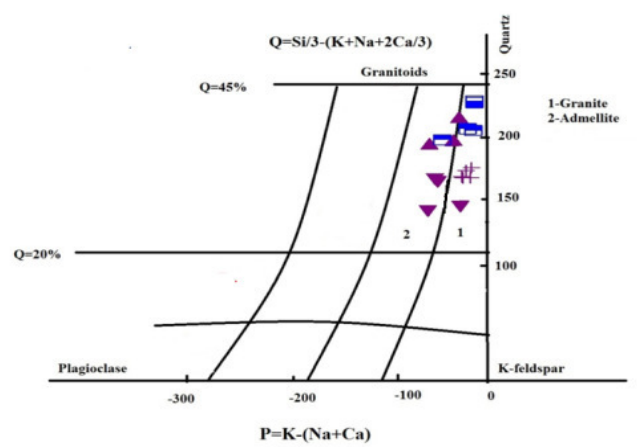

Fig.38:P-Q diagram (Debon and Fort, 1988)

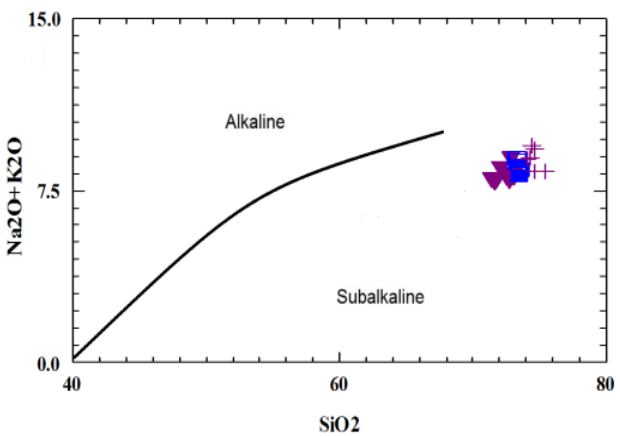

Fig.39: $\left(\mathrm{Na}_{2} \mathrm{O}+\mathrm{K}_{2} \mathrm{O}\right)$ versus $\mathrm{SiO}_{2}$ diagram (Irvine and Baragar ,1971)

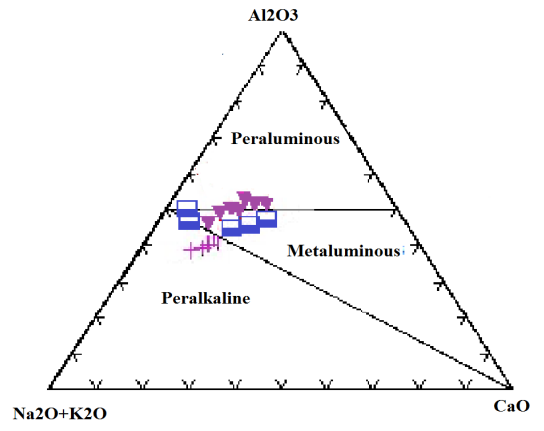

Fig.40: $\mathrm{Al}_{2} \mathrm{O}_{3}-\mathrm{CaO}-\left(\mathrm{Na}_{2} \mathrm{O}+\mathrm{K}_{2} \mathrm{O}\right)$ ternary diagram (Shand,1951)

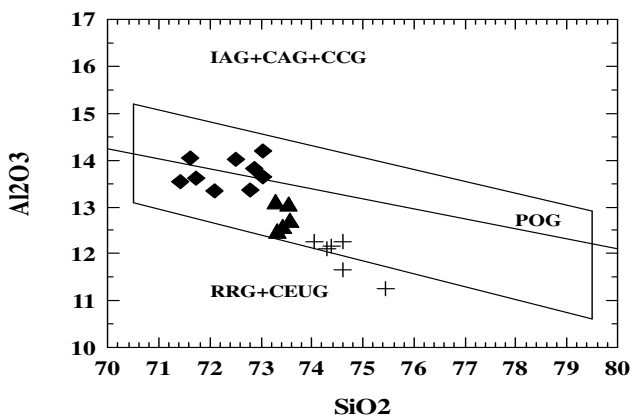

Fig.41:Tectonic setting of the older and younger granites of $\mathrm{W}$. Murra using the modal analyses (Maniar and Piccoli, 1989)

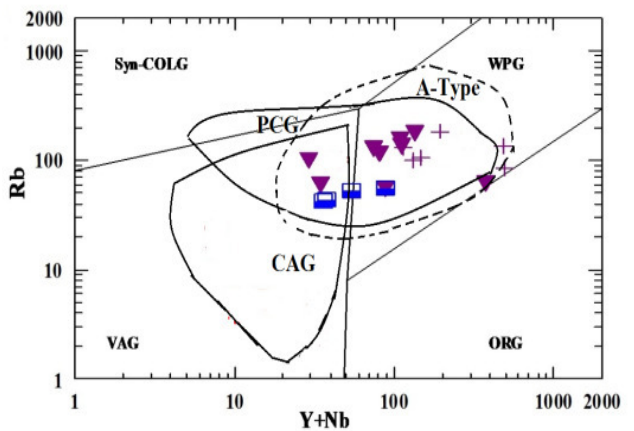

Fig.42:Discrimination diagram for tectonic setting of W. Murra granites (Pearce et al., 1984) 


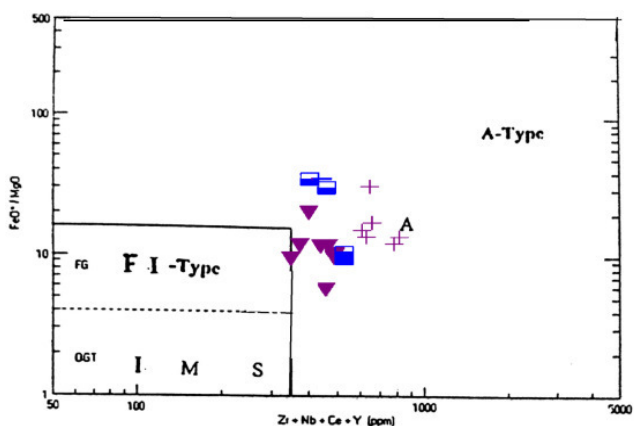

Fig. 43: $\mathrm{FeO} /(\mathrm{MgO}-\mathrm{Zr}+\mathrm{Nb}+\mathrm{Y}+\mathrm{CeO})$ (Whalen et al.,1987)

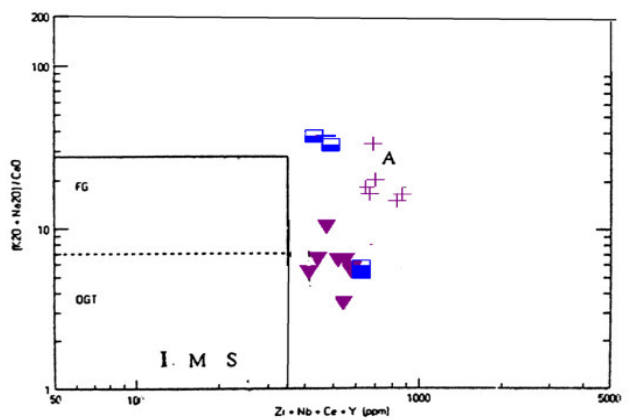

Fig.44: $\left(\mathrm{Na}_{2} \mathrm{O}+\mathrm{K}_{2} \mathrm{O}\right) / \mathrm{CaO}$ diagram (Whalen, et al.,1987)

in $A_{2}$ subgroup of Eby (1992), (Fig.45). This means that the studied granites emplaced in a variety of tectonic settings including post collisonal granite environment and also, A2 group has apparent crustal source. The ferroan to weakly magnesian character and the overall alkalic to alkalicalcic affinities of most rock types (Fig.45) are consistent with the original definition of A-type granitoids proposed by Loiselle and Wones (1979) and as emphasized by Frost and Frost (2011). Traditionally the syenogranites have been assigned to a calc-alkaline granite suite (e.g., Harris et al., 1986), but their compositions plot in the field of alkaline to alkali-calcic magmas (not shown). This evidence suggests an important petrogenetic connection between the different

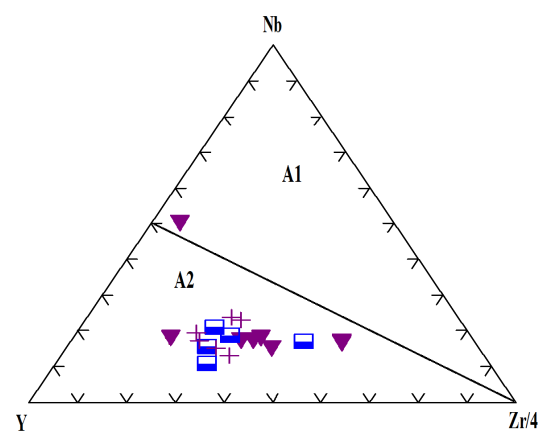

Fig.45:Nb-Y-Zr/4 ternary diagram (Eby et. Al., 1992)

A-type granitic rocks. In element variation diagrams (not shown), the Wadi Murra granitic rocks show consistent trends and systematic variations without a compositional gap between the monzogranites, syenogranites and the alkali feldspar granites. Such geochemical variations indicate that these granites are genetically related.

The REE abundances in Wadi Murra granites are shown in Table (1). Among the studied granites, the syenogranite and alkali feldspar granite display higher average $\Sigma$ REEs contents (423 and $300 \mathrm{ppm}$, respectively) as compared with the world average of granitic rocks $(250 \mathrm{ppm})$ given by Hermann (1974) and with the monzogranite (214 ppm).

$(\mathrm{La} / \mathrm{Yb})_{\mathrm{N}},(\mathrm{La} / \mathrm{Sm})_{\mathrm{N}}$ and $(\mathrm{Gd} / \mathrm{Lu})_{\mathrm{N}}$ ratios are used as a measure of the degree of fractionation of REEs, LREEs and HREEs respectively. The Eu anomaly is estimated as $\left(\mathrm{Eu} / \mathrm{Eu}^{*}\right)_{\mathrm{N}}$ ratio (Table 2).

The chondrite-normalized REEs distribution patterns of Wadi Murra granites, using the values of Taylor and McLennan (1985), show negative slope with relative enrichment in the LREEs compared to the HREEs. The LREEs have high degree of fractionation while the HREEs have limited degree of fractionation. They exhibit negative Eu-anomaly. The younger granites of Wadi Murra show strong negative Eu-anomalies (Fig.46). 
Table 2: Some ratios of the rare earth elements in younger granites

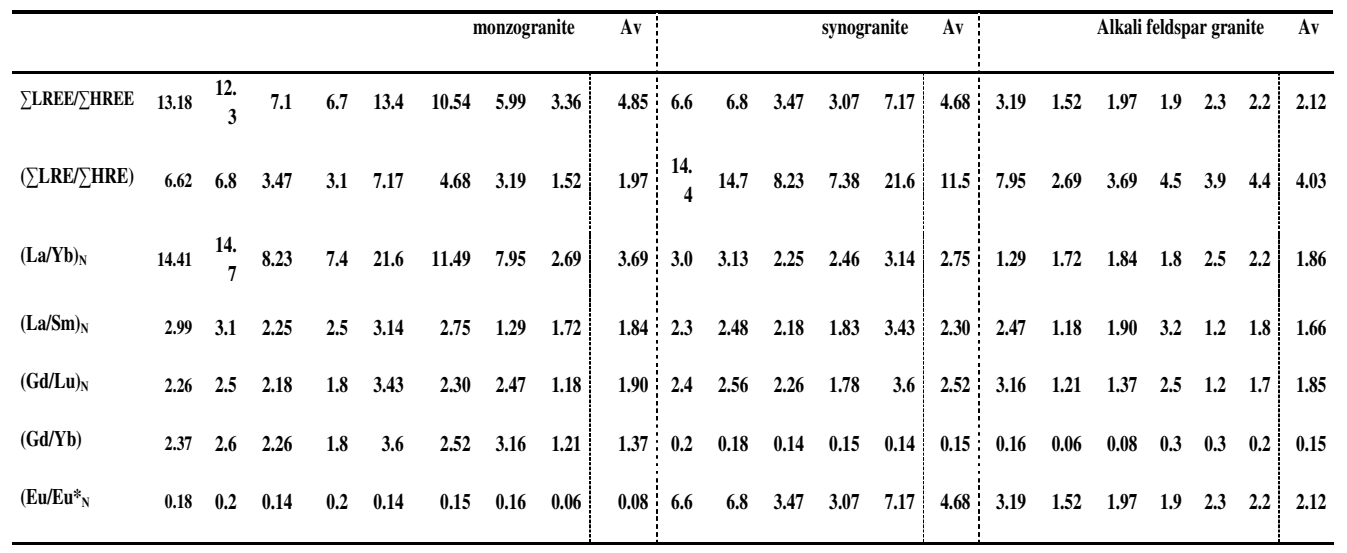

Schnetzler and Philpotts (1970) stated that the uptake of $\mathrm{Eu}^{+2}$ by plagioclase depends upon the anorthite content (since $\mathrm{Eu}^{+2}$ substitutes for $\mathrm{Ca}$ in the plagioclase structure). This would reduce the Eu content of the more sodic plagioclase and consequently the more fractionated rocks. In the studied granites the $(\mathrm{Eu})_{\mathrm{cn}}$ content decreases gradually from monzogranites and reaches the lowest value in the alkali feldspar granites (about 0.15). Figure (46) shows that the normalized REE distribution patterns for the younger granites of Wadi Murra area are the same. This reveals that the studied granites are derived from the same magma. The increase in the total concentration of REEs, $\left(\sum \mathrm{LREE} / \sum \mathrm{HREE}\right)_{\mathrm{N}}$ and $(\mathrm{La} /$ $\mathrm{Yb})_{\mathrm{N}}$ in syenogranites relative to alkali feldspar granites and monzogranites may due to ascending hydrothermal fluids.

The average $(\mathrm{La} / \mathrm{Yb})_{\mathrm{cn}}$ ratio (which represents the measure for LREEs / HREEs) for the younger granites (average value 6.91). Generally, this is the direction of increasing differentiation, because the HREEs (smaller cations) prefer entry to the earlier formed crystals leaving the residual melts progressively enriched in LREEs (larger cations). Thus, the highly fractionated rocks have higher LREEs relative the HREEs contents. The increase in the total concentration of REEs in syenogranites relative to alkali feldspar granites and monzogranites is a very good evidence for ascending hydrothermal fluids. The alkali feldspar granite show the highest value of HREEs that the causes of low value of LREEs / HREEs.

Chondrite-normalized REE patterns of (Taylor and McLennan, 1985) of the considered granites (Fig.46) display a gull-wing shape characterized by large to moderately fractionated patterns and high LREE relative to the HREE contents.

The average $\sum$ REE value of the monzogranite is $213.49 \mathrm{ppm}$. It displays enrichment in LREE (181.93) with HREE (31.56). The monzogranite has REE pattern remarkably shows strong negative Eu anomaly (ranging 0.03 to 0.57 ) and slightly positive Ce anomaly (ranging from 11.57to 196.4). The normalized average ratio show $(\mathrm{La} / \mathrm{Yb})_{\mathrm{N}}$ ratio (ranging from 0.81 to 22.77$)$ and $(\mathrm{La} / \mathrm{Sm})_{\mathrm{N}}$ ratio (ranging 1.29to 3.08) with $(\mathrm{Gd} / \mathrm{Yb})_{\mathrm{N}}$ average ratio (ranging 0.33to 3.42), (Table 1).

The syenogranite and alkali feldspar granite have high REE abundances ( $\sum \mathrm{REE}=423.2$, 299.8). The average LREE concentration of the syenogranites (382.9) were usually high- 


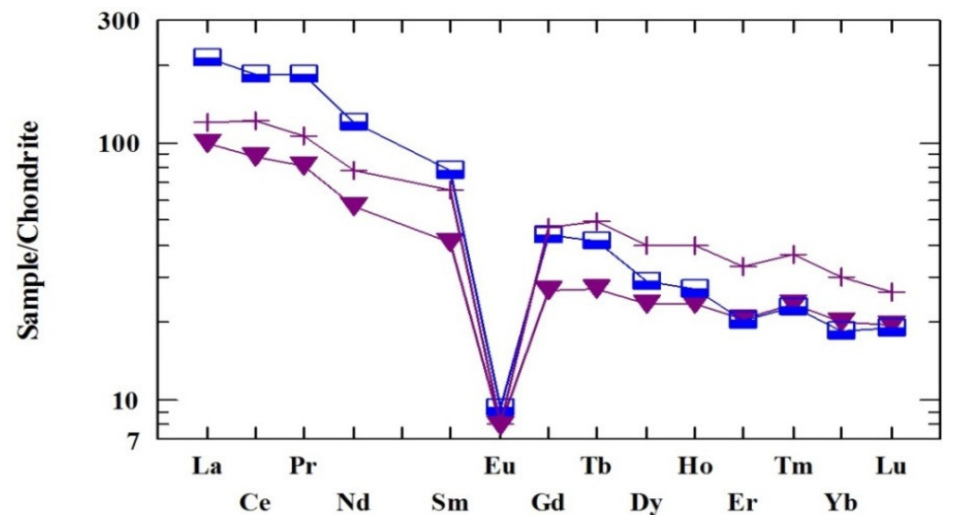

Fig.46:Normalized REE patterns of the younger granites of Wadi Murra area (After Taylor and McLennan, 1985)

er than the average concenteration of HREE (40.3). The syenogranites exhibit REE pattern characterized by a steep negative slope from $\mathrm{Ce}$ to Eu, apartially or nearly flat HREE signature, a high negative Eu anomaly ( 0.140.18 ) and slightly a negative Ce anomaly (151.5-207.3). The syenogranite has an average $(\mathrm{La} / \mathrm{Yb})_{\mathrm{N}}$ ratios (ranging from7.38 to $21.56)$ and $(\mathrm{La} / \mathrm{Sm})$ ratio (ranging from 2.25 to 3.14) with $(\mathrm{Gd} / \mathrm{Yb})_{\mathrm{N}}$ average ratio (Table $1)$.

The average $\sum$ REE value of the alkali feldspar granite is $299.8 \mathrm{ppm}$. It displays enrichment in LREE (246) with HREE (53.77). The alkali feldspar granite have REE pattern remarkably shows more or less closely similar behavior to that of the most primitive granite of the syenogranite but with strong Eu anomaly (ranging from 0.06 to 0.26 ) and slightly positive Ce anomaly (25.29-200). The normalized average ratio show $(\mathrm{La} / \mathrm{Yb})_{\mathrm{N}}$ ratio (ranging from 2.69 to 7.95$)$ and $(\mathrm{La} / \mathrm{Sm})_{\mathrm{N}}$ ratio (ranging from 1.29 to 2.45$)$ with $(\mathrm{Gd} / \mathrm{Y} \mathrm{b})_{\mathrm{N}}$ average ratio (ranging 1.15 to 3.16 ), (Table 1 ).

\section{Petrogenetic Significance Of The REEs Patterns}

There are some constraints concerning the petrogenetic processes responsible for the evolution of the studied rock. This demands the prediction of whether the whole granitic rock sequences of Wadi Murra area had evolved from monzogranites to alkali-feldspar granites from chemically similar magma source regions. The application of $\mathrm{Al}_{2} \mathrm{O}_{3} / \mathrm{TiO}_{2}-\mathrm{TiO}_{2}$ diagram (Fig.47) suggested by Sun and Nesbitt (1978) indicates that the studied granitic rocks resulted from a continuity of magma evolution. The high $\mathrm{TiO}_{2}$ content characterize the least fractionated varieties (monzogranite), while the lower $\mathrm{TiO}_{2}$ content represents the more fractionated varieties (syenogranites and alkali- feldspar granites).

The correlations among major oxides and trace elements refer to the strong associations of strongly incompatible elements such as the HFSE, Y, Ga and $\mathrm{Rb}$ with $\mathrm{SiO}_{2}$. The negative correlation between $\mathrm{Sr}$ and $\mathrm{SiO}_{2}$ suggests that, the various rock units are controlled by magmatic differentiation processes. The REEs patterns indices are used to characterize the petrogenetic significance of the previously concluded diagnostic features of source region and magma evolution history. Generally, the REEs patterns of a cogenetic rock series contain variable geochemical tracers that provide geochcmical information on their origin and petrogenetic history (Takahashi et al., 2000). The REE content, variation trends and degree of fractionation throughout igneous 


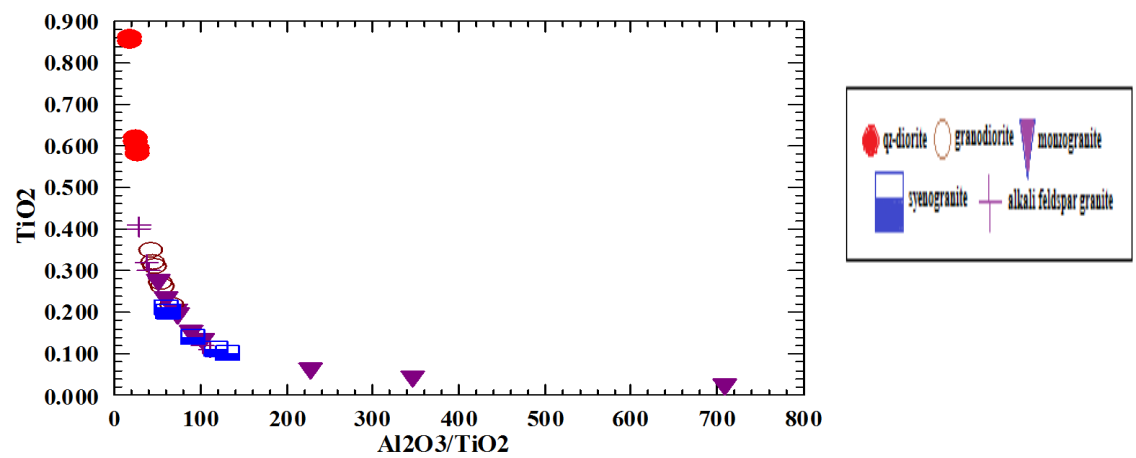

Fig.47: $\mathrm{Al}_{2} \mathrm{O}_{3} / \mathrm{TiO}_{2}-\mathrm{TiO}_{2}$ diagram of the studied granitoid rocks at Wadi Murra area South Eastern Desert (Sun and Nesbitt, 1978)

series are genetic fundamentals in interpreting the chemical composition varieties (Zou and Zindler 1996; Zou 1998 \& 2000).

The REEs indices of the investigated three granitic rock units are plotted against the differentiation index of the magmatic system (e.g $\mathrm{SiO}_{2}$ ). The petrogenetic significance of the REEs content, degree of fractionation and trends and the pattern anomalous throughout the system is illustrated in the following remarks:

1-The variation in $\sum R E E$ and fractionation trends as measured by the ratios $(\mathrm{La} / \mathrm{Yb})_{\mathrm{N}}$, $(\mathrm{La} / \mathrm{Sm})_{\mathrm{N}}$ and $(\mathrm{Gd} / \mathrm{Yb})_{\mathrm{N}}$, provide evidences for telescoping into single comagmatic differentiated series. The REEs content shows progressive constructive enrichment reaching highest content in syenogranites and alkali feldspar granites (Fig.48a). The least differentiated rock unit, monzogrante, has fair degree of fractionation trend [" $\mathrm{r}$ " ratio $>1 ; \quad(\mathrm{La} / \mathrm{Yb})$ $>1$, (Fig. 48b). These points to limited crustal contamination of mantle melt source having MORB proportions composition. The progressive uniform fractionation trends throughout the series (Fig.48b) refer to normal magmatic fractional crystallization processes controlling their petrogenetic history. The modal involves distinctive feldspar fractionation signatures throughout the series such as LREEs content and fractionation trend (Figs.48a,b); $\mathrm{Eu} / \mathrm{Eu}^{*}, \mathrm{Ca}^{2+}$ and $\mathrm{Sr}^{2+}$ systematic inter-element relation (Fig.48c). In addition, the systematic REEs geochemical behavior (Figs.48a,b\&c) rule out extensive alteration and large scale crustal contamination and/or magma mixing, since dramatic variation changes is not evident throughout the petrogenetic history of the rock unit series. Moreover, each rock unit of the series carries its own REEs geochemical signature inventory referring to mantle melt source of a co-magmatic REEs patterns molded by fractional processes.

2-The fractionation trends $(\mathrm{Sm} / \mathrm{Ho})$ show consistent variation with neighboring subgroups (Figs.48a\&b). Such conformable geochemical behavior subgroups refer to their coherent behavior in magmatic differentiation sequence and necessitate two requirement :1) subordinate role of amphibole fractionation, which has preference incorporation of MREEs with convex upward fractionation trend (maximum at Ho) in monzogranite to alkali feldspar granite system (Hilyard et al., 2000) and 2) Absence of amphibolized peridotites contaminant in monzogranite or amphibole-bearing curmulate xenoliths throughout the genetic history of the series. Such effects are generally abundant in oceanic arc, continental crust and the partial melting products of these rocks (Rapp, 1995). 

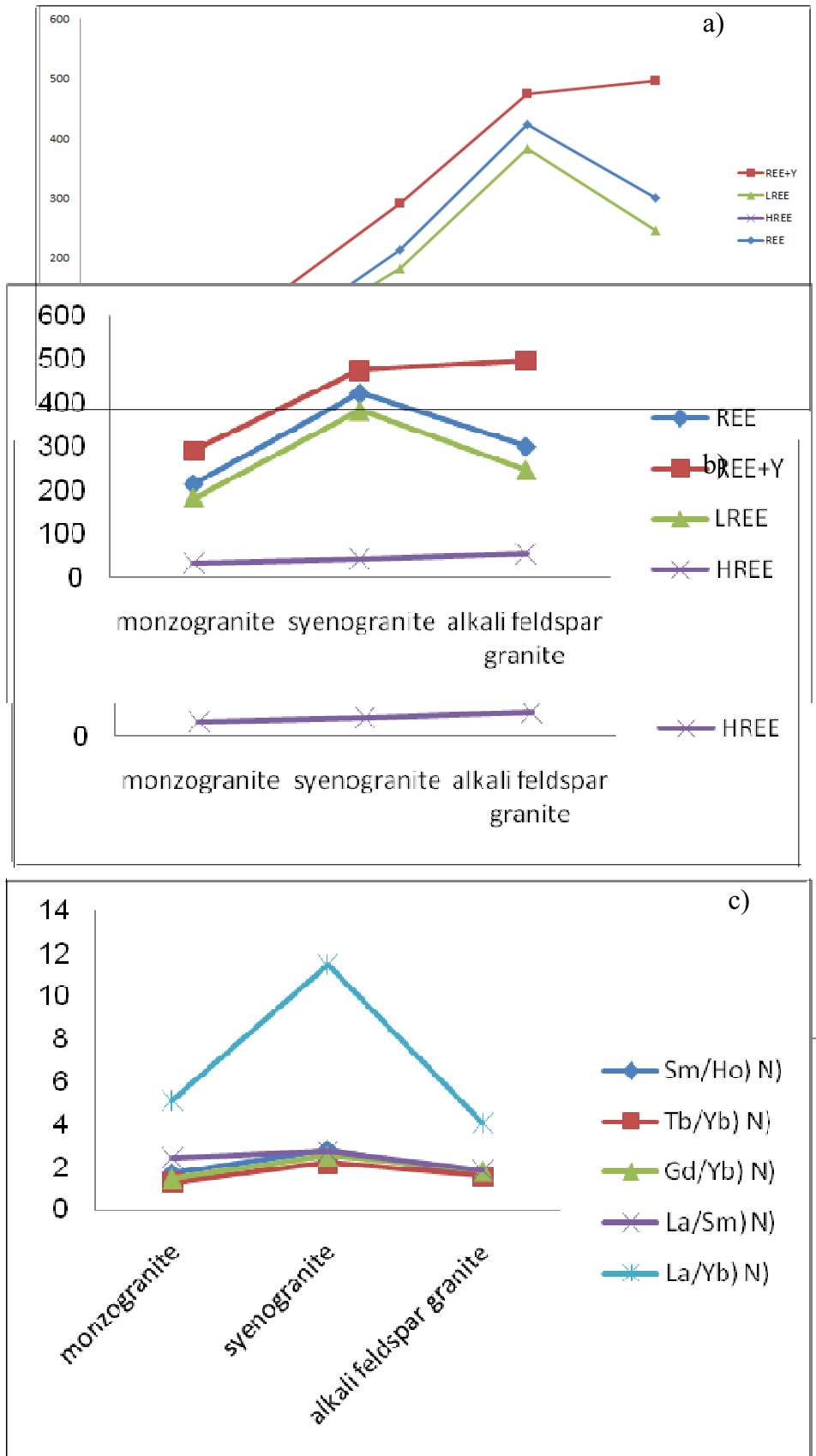

Figs.48a,b,c:The REEs variation pattern of the studied granitoids rocks at Wadi Murra area South Eastern Desert, Egypt 
3-In igneous environments (relative reducing conditions) europium is almost entirely present as divalent state $\left(\mathrm{Eu}^{2+}\right)$. So, unlike the rest of the REEs $\left(\operatorname{Ln}^{3+}\right)$, which are mainly associated with accessory minerals (Panahi et al., 2000), $\left(\mathrm{Eu}^{2+}\right)$ partitions 20 to 100 times more efficiently in plagioclase (Wilke and Behrens, 1999) replacing $\mathrm{Ca}^{2}, \mathrm{Sr}^{2+}$ and $\mathrm{Na}^{+}$ (Henderson, 1996). This anomalous behavior is of particular interest which makes "Eu" acts as a sensitive indicator for destruction of plagioclase structure (since it does not reside in sausurite, sericite or illite structure ), $\mathrm{Pa}$ nahi et al., (2000). The regular uniform concomitant relationship of $\mathrm{Eu} / \mathrm{Eu}^{*}, \mathrm{Ca}^{2+}$ and $\mathrm{Sr}$ ${ }^{2+}$ during the differentiation sequence of the system (Fig.48c) clearly refers to the limited alteration effect of plagioclase. Otherwise, the effect will yield to dramatic negative Eu anomaly associated with similar behavior of $\mathrm{Ca}, \mathrm{Sr}, \mathrm{K}$ and $\mathrm{Na}$. Furthermore, Nagasawa and Schnetzler, (1971) measured $\mathrm{Eu}$ and $\mathrm{Sr}$ distribution in coexisting $\mathrm{Ca}$ and $\mathrm{K}$-feldspars and concluded that $\mathrm{Eu}^{+2}$ behaves very similar much like $\mathrm{Sr}^{2+}$. The reported similar uniform geochemical behavior of the $\mathrm{Eu}^{+2}$ and $\mathrm{Sr}^{2+}$ during magmatic differentiation sequence (Fig.48c) confirm the limited transformation (chemical weathering and/or alteration) of the rock forming $\mathrm{Ca}$ and $\mathrm{K}$-feldspars in to accessory phases throughout the monzogranite- alkali feldspar granite system.

4-The linear variation of " $r$ "ratios $(\mathrm{La} / \mathrm{Yb})_{\star}$, $\sum$ LREEs and $\sum$ HREEs during differentiation sequence (Figs.48a\&b) refer to the absence of intensive effect of accessory minerals characterized by appreciable preference incorporation of certain REEs subgroup such as allanite, xenotime, garnet, sphene, monazite and zircon (Henderson, 1984; Hanson, 1989 and Evans and Hanson, 1993). The variations of REEs indicate that they were ordered according to magma fractionation processes.

5-The REEs patterns show that, the elements maintain their geochemical coherent behavior during the whole differentiation sequence of the system (vis., parallelism of the patterns and straight forward behavior of the indices (Fig.48a). So, such systematic can be modelled according to the coherent geochemical behavior of the rare earth elements and not to the stability constants of their complexes. The latter have different geochemical systematic, depending upon the ligand varieties that yield different fractionation trend of the REEs patterns throughout the system. So the system was deficient in metal ligand free radicals such as $\mathrm{O}^{2-}, \mathrm{OH}^{-}, \mathrm{F}^{-}, \mathrm{CO}_{3}^{-2}, \mathrm{SO}_{4}{ }^{2-}, \mathrm{PO}^{3-}$ etc and hence the system is non-mineralized. Since such metal ligand volatile agents act as the main role in ore genesis (via, mobilization, concentration and transportation of metallogenic metals and ore deposition as result of degassing (decomplexing) of the ore-bearing hydrothermal precipitation system).

\section{REE Tetrad Effect}

The term tetrad effect is refered to the split of chondrite-normalized REEs patterns into four rounded segments called tetrads (first tetrad, $\mathrm{La}-\mathrm{Nd}$, second tetrad $\left(\mathrm{P}_{\mathrm{m}}\right)-\mathrm{Gd}$, third tetrad, Gd-Ho, fourth tetrad, Er-Lu). The rounded segments are either convex or concave and form M-shaped and $\mathrm{W}$-shaped lanthanide distribution patterns (Masuda et al., 1987). The "tetrad" effect is found in the rare earth elements $(\mathrm{La}-\mathrm{Lu})$ and results from increased stability at quarter, half, three-quarter, and complete filling of the $4 f$ electron shell (McLennan, 1994). Tetrad effect-like REEs patterns are reported both in magmatic rocks and precipitates from hydrothermal fluids. Recent discussions about the tetrad effect focus on highly evolved igneous rocks (Bau, 1996). Ireber (1999) and Monecke et al., (2002) introduced procedures for quantification of tetrad effect and individual tetrads.

The tetrad effect in lanthanide patterns of whole-rock samples was quantified by Eqns. 1, 2 and 3 proposed by Irber (1999). The calculated sizes $t_{1}, t_{3}$ and $T$ of the tetrad effect are listed in Table (3).

The younger granites show a pattern with 
convex first tetrad effect $\left(t_{1}=1.4-1.6\right)$ while the calculated sizes $t_{3}$ and $T$ of the tetrad effect are below the level of significance (1.1).

The late stage fluid-melt interaction possibly affected the size of the tetrad effect of individual tetrads (Jahn et al., 2001). Fluid-melt interaction in the late stage of fractional crystallization is suggested to be the most important factor controlling the formation of REE tetrad effects in the granites (Zhenhua et al. 2002). Curved segments in normalized whole rock REE patterns can be introduced during hydrothermal fluid-rock interaction (Monecke et al., 2007). This may have caused the first tetrad $\left(\mathrm{t}_{1}\right)$ in the Wadi Murra younger granites.

\section{RADIOACTIVITY}

\section{Distribution of The Radioelements In Younger Granites}

The radiometric data of the radioelements for monzogranite show a wide variation in $\mathrm{eU}$ and $\mathrm{eTh}$ contents. The eU content ranges from 6 to $12 \mathrm{ppm}$ with an average of $8.5 \mathrm{ppm}$ and the eTh content ranges from 5 to $16 \mathrm{ppm}$ with an average of $10.12 \mathrm{ppm}$. The potassium content is ranging between $3.08 \%$ and $7.67 \%$ with an average of $4.49 \%$ (Table 3). The eU content ranges from 2.4 to $6 \mathrm{ppm}$ with an average of $3.78 \mathrm{ppm}$ and the eTh content ranges from 7 to $9 \mathrm{ppm}$ with an average of $8 \mathrm{ppm}$. The potassium content is ranging between $4.08 \%$ and $4.69 \%$ with an average of 4.53 $\%$ (Table 4). The eU content ranges from 5 to $9.8 \mathrm{ppm}$ with an average of $6.86 \mathrm{ppm}$ and the eTh content ranges from 8 to $16 \mathrm{ppm}$ with an average of $13.25 \mathrm{ppm}$. The potassium content is ranging between $4.9 \%$ and $4.95 \%$ with an average of $4.70 \%$ (Table 4 ).

Darnely (1982) concluded that the uraniferous granites is any granitic mass containing $\mathrm{U}$ at least twice Clark value $4.0 \mathrm{ppm}$ for normal granites. Monzogranite have eU content ranges from 6 to $12 \mathrm{ppm}$ with an average of $8.5 \mathrm{ppm}$ and Alkali feldspar granite have eU content ranges from 5 to $8 \mathrm{ppm}$ with an average of 6.86ppm.

The anomalous samples were taken from the northing part of the studied area, these samples classified as syenogranite and alkali

Table 3:Tetrad effect analyses for older and younger granites of Wadi Murrah area

\begin{tabular}{|c|c|c|c|c|c|c|c|}
\hline No. & Rock types & $\mathbf{t 1}$ & Avg & $\mathbf{t 3}$ & Avg & $\mathbf{T}$ & Avg \\
\hline G32 & \multirow{9}{*}{ monzogranite } & 0.94 & \multirow{9}{*}{1.16} & 1.07 & \multirow{9}{*}{1.09} & 1.01 & \multirow{9}{*}{1.08} \\
\hline G45 & & 1.14 & & 0.87 & & 0.96 & \\
\hline G43 & & 1.13 & & 1.01 & & 1.07 & \\
\hline G47 & & 1.16 & & 1.03 & & 1.09 & \\
\hline G48' & & 1.14 & & 1.14 & & 1.06 & \\
\hline G48'" & & 1.35 & & 1.35 & & 1.11 & \\
\hline G50 & & 1.18 & & 1.24 & & 1.21 & \\
\hline G51' & & 1.25 & & 1.1 & & 1.17 & \\
\hline G57' & & 1.16 & & 1.04 & & 1.08 & \\
\hline $\mathbf{6 5 5}$ & \multirow{5}{*}{ syenogranite } & 1.13 & \multirow{5}{*}{1.16} & 0.93 & \multirow{5}{*}{1.01} & 1.02 & \multirow{5}{*}{1.08} \\
\hline G55' & & 1.26 & & 0.94 & & 1.09 & \\
\hline G58 & & 1.17 & & 1.04 & & 1.1 & \\
\hline G58' & & 1.16 & & 1.15 & & 1.16 & \\
\hline G60 & & 1.09 & & 0.89 & & 0.98 & \\
\hline $\mathbf{G} 31$ & \multirow{6}{*}{$\begin{array}{r}\text { Alkali } \\
\text { feldspar } \\
\text { granite }\end{array}$} & 1.07 & \multirow{6}{*}{1.14} & 1.15 & \multirow{6}{*}{1.04} & 1.11 & \multirow{6}{*}{1.08} \\
\hline G59 & & 1.19 & & 1.07 & & 1.13 & \\
\hline G62' & & 1.31 & & 1.05 & & 1.17 & \\
\hline G63 & & 0.95 & & 1.06 & & 1 & \\
\hline G64 & & 1.15 & & 0.94 & & 1.04 & \\
\hline G65 & & 1.14 & & 0.97 & & 1.05 & \\
\hline
\end{tabular}


Table 4:Analyses of the $\mathrm{U}$ (ppm), eU(ppm) and D-factor contents of the younger granites

\begin{tabular}{|c|c|c|c|c|c|c|c|}
\hline $\begin{array}{l}\text { S.No } \\
\text { Rock Ui }\end{array}$ & its & $\mathbf{U}$ & AVG & $\mathrm{eU}$ & AVG & $\begin{array}{r}\text { D- } \\
\text { factor }\end{array}$ & AVG \\
\hline G32 & \multirow{8}{*}{ 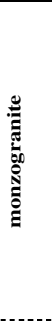 } & 2.52 & \multirow{8}{*}{4.59} & 7 & \multirow{8}{*}{8.5} & 0.36 & \multirow{8}{*}{0.54} \\
\hline G45 & & 2.5 & & 6 & & 0.41 & \\
\hline G47 & & 8 & & 12 & & 0.66 & \\
\hline G48 & & 4.5 & & 10 & & 0.45 & \\
\hline G48" & & 3.2 & & 8 & & 0.4 & \\
\hline G50 & & 6.2 & & 7 & & 0.88 & \\
\hline G51 & & 5.6 & & 12 & & 0.46 & \\
\hline G57' & & 4.2 & & 6 & & 0.7 & \\
\hline G55 & \multirow{5}{*}{ 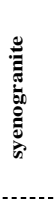 } & 2.5 & \multirow{5}{*}{2.34} & 6 & \multirow{5}{*}{3.78} & 0.41 & \multirow{5}{*}{0.69} \\
\hline G58 & & 2.4 & & 4 & & 0.6 & \\
\hline G55/ & & 2.5 & & 2.5 & & 1 & \\
\hline G58/ & & 2.4 & & 2.4 & & 1 & \\
\hline G60 & & 1.9 & & 4 & & 0.47 & \\
\hline G31 & \multirow{6}{*}{ 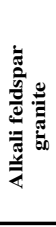 } & 5.2 & \multirow{6}{*}{6.65} & 5 & & 1.04 & \multirow{6}{*}{0.96} \\
\hline G59 & & 5.4 & & 8 & & 0.67 & \\
\hline G62/ & & 4.8 & & 5.4 & 6.86 & 0.88 & \\
\hline G63 & & 9.8 & & 5 & & 1.96 & \\
\hline G64 & & 9.8 & & 9.8 & & 1 & \\
\hline G65 & & 49 & & 8 & & 0.61 & \\
\hline
\end{tabular}

feldspar granite. The results of the analyzed $\mathrm{U}$, The and $\mathrm{Th} / \mathrm{U}$ ratios are presented in Table (5).

The $U$ contents in the studied syenogranites anomalous sites ranges from 43 to 133 ,while the Th contents anomalous sites ranges from 55 to 156 . The $\mathrm{Th} / \mathrm{U}$ ratios ranges from 0.70 to 2.64 .

The U contents in the studied alkali feldspare granites anomalous sites ranges from 141 to 319 , while the Th contents anomalous sites ranges from 60 to 284 . The $\mathrm{Th} / \mathrm{U}$ ratios ranges from 0.05 to 0.89 .

The relation between $\mathrm{U}$ and $\mathrm{Th}$ contents (ppm) among the anomalous sites through the granitic rocks of Wadi Murra is examined by plotting The $\mathrm{U}$ contents against $\mathrm{Th}$ contents (Fig.49). It show strong negative correlation between both $U$ and Th. This relation between $\mathrm{U}$ and $\mathrm{Th}$ indicate their enrichment of
Table 5:Chemical analyses of the $U$ (ppm) and Th (ppm) contents of the anomalous granites of Wadi Murra area

\begin{tabular}{|c|c|c|c|c|}
\hline$\overline{\mathrm{S.No}}$ & & $\begin{array}{r}\mathbf{U} \\
(\mathbf{p p m})\end{array}$ & $\begin{array}{r}\text { Th } \\
(\mathbf{p p m})\end{array}$ & $\mathbf{T h} / \mathbf{U}$ \\
\hline 1 & & 43 & 115 & 2.64 \\
\hline 2 & 象 & 65 & 92 & 1.41 \\
\hline 3 & สี & 78 & 55 & 0.70 \\
\hline 4 & 80 & 91 & 104 & 1.14 \\
\hline 5 & 逍 & 133 & 156 & 1.17 \\
\hline 6 & & 170 & 94 & 0.55 \\
\hline 7 & సี & 165 & 73 & 0.45 \\
\hline 8 & $\bar{n} \frac{\bar{n}}{2}$ & 141 & 60 & 0.42 \\
\hline 9 & 巳 $\Xi$ & 250 & 65 & 0.05 \\
\hline 10 & $\begin{array}{l}\text { 馬 } \\
\text { 美 }\end{array}$ & 319 & 284 & 0.89 \\
\hline
\end{tabular}

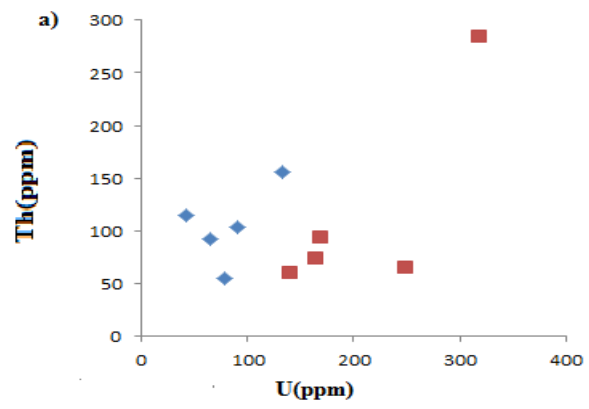

Fig.49:Binary relations between U-Th, for the studied anomalous granites of Wadi Murra area

U relative to Th. on the other hand, it shows strong negative correlation between both the $\mathrm{U}-\mathrm{Th} / \mathrm{U}$ variation diagram, (Fig.50) and Th$\mathrm{Th} / \mathrm{U}$ (Fig.51) show positive correlation. This means that the $\mathrm{Th} / \mathrm{U}$ in the anomalous sites clear enrichment of uranium relative to thorium which may be attributed to the presence of accessory minerals or to the secondary process played main role in uranium enrichment which means that uranium may be added to these granitic sites post magmatically. 


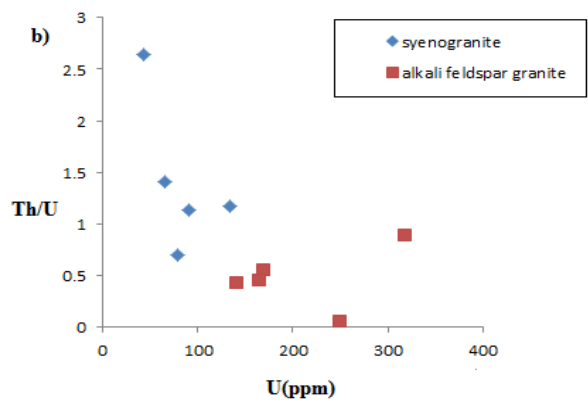

Fig.50:Binary relations between $\mathrm{U}-\mathrm{Th} / \mathrm{U}$ for the studied anomalous granites of Wadi Murra area

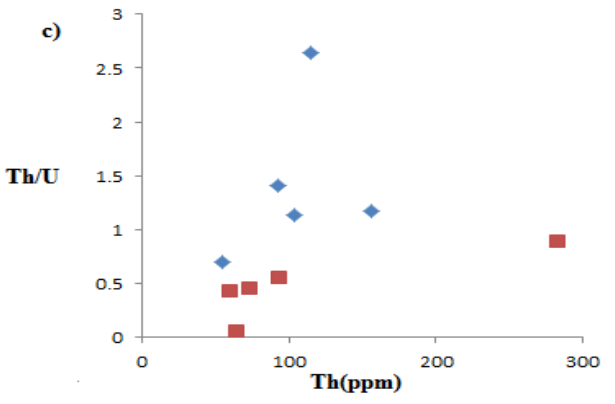

Fig.51:Binary relations between $\mathrm{Th}-\mathrm{Th} / \mathrm{U}$ for the studied anomalous granites of Wadi Murra area

The picked minerals are identified confirmed by environmental Scan Electron Microscope (ESEM) and XRD techniques at laboratories of Nuclear Materials Authority (NMA). The identified minerals in the studied areaare classified to: 1) Secondary uranium minerals (uranophane, autunite and metautunite), 2) Thorium minerals (uranothorie), 3) Niobium - tantalum minerals (columbite), 4) Base metals minerals (pyrite) and 5). Accessory minerals bearing $\mathrm{U}, \mathrm{Th}$ and REEs (zircon, fluorite, monazite, xenotime, sphene, allanite, apatite and iron oxides).

\section{SUMMARY AND CONCLUSION}

Wadi Murra granite is located at south Eastern Desert of Egypt, about $250 \mathrm{~km}$ from Aswan City at the northern side of Wadi Allaqi-Wadi Murra junction. The younger granites at Wadi Murra are classified as monzogranites, syenogranite and alkali feldspar granite. Monzogranite and syenogranie consist mainly of potash feldspar, plagioclase, quartz and mafic minerals with noticeable amount of opaque minerals. The main characteristic texture is equigranular with medium to coarse grain size. Alkali-feldspar granite is medium grained and characterized by hypidiomorphic granular texture and composed mainly of alkali feldspar (orthoclase and minor microcline), quartz and subordinate plagioclase. The main mafic minerals are either amphibole or biotite. Accessories are represented by zircon, sphene, apatite and iron oxides. Geochemicaly they are calc-alkaline in nature, metaluminous to peralkaline magma, and emplaced in a within plate granite environmed and have $\mathrm{A}_{2}$ type, which means that these granites emplaced in post collisional granite environment and also, $\mathrm{A}_{2}$ group has apparent crustal source.

The radiometric data for monzogranite shows a wide variation in $\mathrm{eU}$ and $\mathrm{eTh}$ contents. The eU content ranges from 6 to 12 $\mathrm{ppm}$ with an average of $8.5 \mathrm{ppm}$ and the eTh content ranges from 5 to $16 \mathrm{ppm}$ with an average of $10.12 \mathrm{ppm}$. The potassium content is ranging between $3.08 \%$ and $7.67 \%$ with an average of $4.49 \%$. The eU content in syenogranite ranges from 2.4 to $6 \mathrm{ppm}$ with an average of $3.78 \mathrm{ppm}$ and the eTh content ranges from 7 to $9 \mathrm{ppm}$ with an average of $8 \mathrm{ppm}$. The potassium content is ranging between $4.08 \%$ and $4.69 \%$ with an average of 4.53 $\%$. The eU content in alkali feldspar granite ranges from 5 to $9.8 \mathrm{ppm}$ with an average of $6.86 \mathrm{ppm}$ and the eTh content ranges from 8 to $16 \mathrm{ppm}$ with an average of $13.25 \mathrm{ppm}$. The potassium content is ranging between $4.9 \%$ and $4.95 \%$ with an average of $4.70 \%$.

The the picked minerals from the anomalous samples are identified confirmed by 
environmental Scan Electron Microscope (ESEM) and XRD techniques at laboratories of Nuclear Materials Authority (NMA). The identified minerals in the studied area are classified to 1) Secondary uranium minerals, (uranophane, autunite and metautunite), 2) Thorium minerals (uranothorite), 3) Niobium - tantalum minerals (columbite), 4) Base metal minerals (pyrite) and 5) Accessory minerals bearing $\mathrm{U}$, Th and REEs (zircon, fluorite, monazite, xenotime, sphene, allanite, apatite and iron oxides).

\section{REFERENCES}

Abdalla, H. M.; Ishihara. S.; Matsueda , H., and Abdel Monem, A.A., 1996. On the albite-enriched granitoids at Umm Ara area. South eastern Desert. Egypt. 1. Geochemical, ore potentiality and fluid inclusions studies,J. Geochem. Exp. ,57,127-138.

Agangi, A.; Kamenetsky, V.S., and McPhie, J., 2010. The role of fluorine in the concentration and transport of lithophile trace elements in felsic magmas: insights from the Gawler Range Volcanics, South Australia. Chemical Geol., 273, 314-325.

Ali, K.A.; Jeon, H.; Andresen, A.; Li, S-Q.; Harbi, H.M., and Hegner, E., 2014. U-Pb zircon geochronology and Nd-Hf-O isotopic systematics of the Neoproterozoic Hadb adh Dayheen ring complex, Central Arabian Shield, Saudi Arabia. Lithos, 206-207, 348-360.

Ali, K.A.; Moghazi, A.M.; Maurice, A.E.; Omar, S.A.; Wang, Q.; Wilde, S.A.; Moussa, E.M.; Manton, W.I., and Stern, R.J., 2012. Composition, age, and origin of the $\sim 620$ Ma Humr Akarim and Humrat Mukbid A-Type granites: no evidence for pre27 Neoproterozoic basement in the Eastern Desert, Egypt. Inter. J. Earth Sciences, 101, 1705-1722.

Ali, K.A.; Stern, R.J.; Manton, W.I.; Kimura, J.I., and Khamees, H.A., 2009. Geochemistry, $\mathrm{Nd}$ isotopes and $\mathrm{U}-\mathrm{Pb}$ SHRIMP dating of Neoproterozoic volcanic rocks from the Central
Eastern Desert of Egypt: new insights into the 750 Ma crust-forming event. Precambrian Research ,171, 1-22.

Anderson, J.L., 1983. Proterozoic anorogenic granite plutonism of North America. Geological Society of America Memoir 161, 133-152.

Audétat, A.; Günther, D., and Heinrich, C.A., 2000. Magmatic-hydrothermal evolution in a fractionating granite: a microchemical study of the Sn-W-F-mineralized Mole Granite (Australia). Geochimica et Cosmochimica Acta ,19, 3373-3393.

Bau, M., 1996. Controls on the fractionation of isovalent trace elements in magmatic and aqueous system : evidence from $\mathrm{Y} / \mathrm{Ho}, \mathrm{Zr} / \mathrm{Hf}$ and lanthanide tetrad effect . Contributions to Mineralogy and petrololgy ,123, $323-333$.

Barker, F.; Wones, D.R.; Sharp, W.N., and Desborogh, G.A., 1975. The Pikes Peak Batholith, Colourado Front Range, and a model for the origin of the gabbroanorthosite-syenite-potassic granite suite. Precambrian Research, 2, 97160.

Be'eri-Shlevin, Y.; Katzir, Y.; Blichert-Toft, J.; Kleinhanns, I.C., and Whitehouse, M., 2010. $\mathrm{Nd}-\mathrm{Sr}-\mathrm{Hf}-\mathrm{O}$ isotope provinciality in the northernmost Arabian-Nubian Shield: implications for crustal evolution. Contributions to Mineralogy and Petrology ,160, 181-201.

Be'eri-Shlevin, Y.; Katzir, Y., and Valley, J.W., 2009a. Crustal evolution and recycling in a juvenile continent: oxygen isotope ratio of zircon in the northern Arabian Nubian Shield. Lithos ,107, 169-184.

Be'eri-Shlevin, Y.; Katzir, Y., and Whitehouse, M., 2009b. Post-collisional tectonomagmatic evolution in the northern Arabian-Nubian Shield (ANS): time constraints from ionprobe $\mathrm{U}-\mathrm{Pb}$ dating of zircon. J. Geol. Soc. London, 166, 71-85.

Charoy, B., and Pollard, P. J., 1989. Albit-rich, silica-depleted Metasomatic rocks at Emuford, Northeast Queensland: Mineralogical, 
geochemical, and fuid inclusion constraints on hydrothermal evolution and Tin mineralization Chequ, J., 1981. Fluorite and barite in Saudi Arabia: Saudi Arabian Deputy Ministry for Mineral Resources, Open File Repor, BRGM OF-01-30, 29 p.

Christiansen, E. H.; Bikun, J. W.; Sheridan, M. F., and Burt, D. M., 1984. Geochemical evolution of topaz rhyolites from the Thomas Range and Spor Mountain, Utah, Am. Mineralogist, 69, 223-236.

Christiansen, E. H.; Sheridan, M. F., and Burt, D. M., 1986. The geology and geochemistry of Cenozoic topaz rhyolites from the western United States, Geol. Soc. America Spec. Paper, 205, 1-82.

Clemens, J.; Holloway, J.R.,and White, A.J.R., 1986. Origin of an A-type granite: experimental constraints. American Mineralogist ,71, $317-324$.

Collins, W.J.; Beams, S.D.; White, A.J., and Chappell, B.W., 1982. Nature and origin of A-type granites with particular reference to southeastern Australia. Contributions to Mineralogy and Petrology, 80, 189-200.

Creaser, R.A.; Price, R.C., and Wormald, R.J., 1991. A-type granites revisited: assessment of a residual-source model. Geology, 19, 163-166.

Cuney, M.; Marignac, C., and Weisbrod, A., 1992. The Beauvoir topaz-lepidolite albite granite (Massif Central France): the disseminated magmatic $\mathrm{Sn}-\mathrm{Li}-\mathrm{Ta}-\mathrm{Nb}-\mathrm{Be}$ mineralization. Economic Geol., 87, 1766-1794.

Darnely, A. G., 1982. Hot granites" some general remarks. In: Uranium in granites(Maurice, Y. J.,Ed.). Geol. Surv. Canada, Paper No. 81 - 23, $1-10$.

Debon, F., and Le Fort, P.,1983. Chemical-mineralogical classification of the common plutonic rocks and associations. Trans. R. Soc. Edinbrugh (Earth Sci.), 73, 135-149.

Deer, W. A.; Howie, R. A. and Zussman J., 1985. Rock forming minerals.Longman Group (FE)
Ltd., Hong Kong, 696 p.

De La Roche, H.; Leterrier,J.; Grandclade, P., and Marchal,M.,1980. A classification of volcanic and plutonic rocks using RI-R2 diagram and major element analyses. Its relationship with current nomenclature, Chem. Geol., 29,183210 .

Drysdall, A. R.; Jackson, N. J.; Ramsay, C. R.; Douch, C. J., and Hackett, A. D., 1984. Rare element mineralization related to Precambrian alkali granites in the northwest Arabian Shield. Economic Geol., 79, 1366-1377.

Eby, G.N., 1992. Chemical subdivision of A-type granitoids: petrogenetic and tectonic implications. Geol., 20, 641-644.

El-Bialy, M.Z., and Hassen, I.S., 2012. The late Ediacaran (580-590 Ma) onset of anorogenic alkaline magmatism in the Arabian-Nubian Shield: Katherina A-type rhyolites of Gabal Ma'ain, Sinai, Egypt. Precambrian Research, 216-219,1-22.

El-Gaby, S. I.; Lists, F. K., and Tahrany, R., 1988. Geology, evolution and metallogenesis of the Pan African belt in Egypt, In: The Pan African belt of northeast Africa and adjacent areas Earth Evol .Sci .Vieweigeh (El-Gaby, S. and Greiling, R. O. ,Eds). Tectonic Evalution and Economic Aspects of Late Proterozoic Orogeny, Braun/ Schweig (Viewegel), 17-70.

Evans, O.C., and Hanson,G.N., 1993.Acessory mineral fractionation of rare-earth element (REE) Abundances in granitoid rocks, Chem. Geol.,110, 69-93.

Eyal, M.; Litvinovsky, B.; Jahn, B.M.; Zanvilevich, A., and Katzir, Y., 2010. Origin and evolution of post-collisional magmatism: Coeval Neoproterozoic calc-alkaline and alkaline suites of the Sinai Peninsula. Chemical Geol., 269, 153-179.

Farahat, E.S., and Azer, M.K., 2011. Post-collisional magmatism in the northern Arabian-Nubian Shield: the geotectonic evolution of the alkaline suite at Gebel Tarbush area, south Sinai, Egypt. 
Chemie der Erde-Geochemistry, 71, 247-266.

Foland, K. A., and Allen, J. C., 1991. Magma sources for Mesozoic anorogenic granites of the White Mountain magma series, New England, U.S.A. Contribution to Mineralogy and Petrology, 109, 195-211.

Frost, C.D., and Frost, B.R., 1997. Reduced rapakivi-type granites: the tholeiite connection. Geology, 25, 647-650.

Frost, C.D.,and Frost, B.R., 2011. On ferroan (Atype) granitoids: their compositional variability and modes of origin. J. Petrology ,52, 39-53.

Genna, A.; Nehlig, P., Le ; Goff, E.; Guerrot, C., and Shanti, M .2002. Proterozoic tectonism of the Arabian Shield. Precambrian Research ,117, 21-4.

Hanson, G.N., 1989. An approach to trace element modeling using a simple igneous system as an example, in the Geology and Geochemistery of Rare Earth Elements. Lipin and McMay, Rev. Min., 21,79-97.

Harris, N. B. W.,1982. The petrogenesis of the alkaline intrusives from Arabia and NE Africa and their implications for within plate magmatism. Tectonophysics, 83, 243-258.

Henderson, P.,1984. Rare Earth Element Geochemistery . Elsevier, Amsterdam, 510p.

Henderson, P.,1996. Rare earth elements, introduction and rivew .In: Rare earth minerals: Chemistery, origin and deposits( Jones, A.P., Wall, F. and Williams, C.T.,Eds.) The Mineralogical Society Series, 1-19.

Hermann, A.G., 1974. Yittrium and lanthnides. In: Handbook of geochemistry(Wedepohl, K.H., Ed.). Berlin,Springer.Veriag, 2, 59-71.

Hilyard, M.; Nielsen, R.L.; Beard, J.S.; Patin,O.; Douce, A., and Blencoe., J.,2000. Experimental determination of the partitioning behavior of rare earth and high field strength elements between pargasitic amphibole and natural silicate melts. Geochim.Cosmochim., Acta, No.6, 64,1103-1120.
Irber, W., 1999. The lanthanide tetrad effect and its correlation with $\mathrm{K} / \mathrm{Rb}, \mathrm{Eu} / \mathrm{Eu}^{*}, \mathrm{Sr} / \mathrm{Eu}, \mathrm{Y} / \mathrm{Ho}$ and $\mathrm{Zr} / \mathrm{Hf}$ of evolving peraluninous granite suites . Geochimica et Cosmochimica , Acta , $63,489-508$.

Irvine, T. N., and Baragar W. R. A., 1971. A guide to the chemical classification of the common volcanic rocks. Can. J. Earth Sci., 8,523-548.

Jahn , B.M.; Wu, F.Y.; Cabdevila, R.; Martineau, F.; Zhao, Z.H., and Wang, Y.X., 2001. Highly evolved juvenile granites with tetrad REE patterns : the Woduhe and Baerzhe granites from the Great Xing' an Mountains NE china. Lithos , 59, $171-198$.

Johnson, P.R., 2003. Post-amalgamation basins of the NE Arabian shield and implications for Neoproterozoic III tectonism in the northern East African orogen. Precambrian Research 123, 321-338.

Johnson, P.R.; Andresen, A.; Collins, A.S.; Fowler, A.R.; Fritz, H.; Ghebrab, W.; Kusky, T., and Stern, R.J. 2011. Late Cryogenian-Ediacaran history of the Arabian-Nubian Shield: a review of depositional, plutonic, structural, and tectonic event in the closing stages of the northern East African Orogen. J. African Earth Sciences, 61,167-232.

Johnson, P.R., and Woldehaimanot, B. 2003. Development of the Arabian-Nubian Shield: perspectives on accretion and deformation in the northern East African Orogen and the assembly of Gondwana. In: Proterozoic East Gondwana: supercontinent assembly and breakup (Yoshida M; Dasgupta S, \& Windley B ,Eds). Geol. Soc. London, Special Publications, 206, 289-325.

Katzir, Y.; Eyal,M.; Litvinovsky, B.A.; Jahn, B.M.; Zanvilevich, A.N.; Valley, J.W.; Beeri, Y.; Pelly, I., and Shimshilashvili, E., 2007. Petrogenesis of A-type granites and origin of vertical zoning in the Katharina pluton, Gebel Mussa (Mt.Moses) area, Sinai, Egypt. Lithos ,95, 208-228.

Küster, D., 2009. Granitoid-hosted Ta mineralization in the Arabian-Nubian Shield: ore deposit types, tectono-metallogenetic setting and petro- 
genetic framework. Ore Geology Reviews, 35, 68-86.

Lehmann, B., 1982. Metallogeny of tin: Magmatic differentiation versus geochemical heritage. Econ. Geol., 77, 50-70.

Lehmann, B., 1987. Tin granites, geochemical heritage, magmatic differentiation. Geologic Rundschau, 76, 177-185.

Lehmann, B., and Mahawat, C., 1989. Metallogeny of tin in central Thailand: A genetic concept. Geology ,17, 426-429.

Linnen, R.L., and Cuney, M., 2005. Granite-related rare-element deposits and experimental constraints on $\mathrm{Ta}-\mathrm{Nb}-\mathrm{W}-\mathrm{Sn}-\mathrm{Zr}-\mathrm{Hf}$ Mineralization. In: Linnen, R.L., Samson,

Litvinovsky, A.A.; Jahn, B.M.; Zanvilevich, A.N.; Saunders, A.; Poulain, S.; Kuzmin, D.V.; Reichow, M.K., and Titov, A.V., 2002. Petrogenesis of syenite-granite suites from the Bryansky Complex (Transbaikalia, Russia): implications for the origin of A-type granitoids magmas. Chem. Geol., 189, 105-133.

Loiselle, M. C., and Wones, D. R, 1979. Characteristics and origin of anorogenic granites. Geol. Soc. Am. Abstr. with progr, 11, 468.

London, D., 1986a. The magmatic-hydrothermal transition in the Tanco rare-element pegmatites: evidence from fluid inclusions and phase equilibrium experiments. Amer. Mineral., 71, 376-395.

London, D., 1986b. Formation of tourmaline-rich gem pockets in miarolitic pegmatites. Amer. Mineral., 71, 396-405.

Maniar, P.D., and Piccoli, P.M., 1989.Tectonic discrimination of granitoids. Geol. Soc. Am. Bull., No.101, 635-643.

McLennan, 1994. The maximal generic number of pure nash equilibria . Social Science Working Paper No. 273, California Instit. Technology.

Mass, R.; McCulloch, M. T.; Campbell, I. H., and Page, R. W., 1987. Sm-Nd isotope systematics in uranium-rare earth element mineralization at the Mary Kathleen uranium mine, Queensland: Econ. Geol., 82, 1805-1826.

Masuda, A.; Kawakami, O.; Dohomoto, Y., and Takenaka, T.,1987.Lanthanide tetrad effects in nature: two mutually opposite types. W and M.Geochem. J, 21, 119-124.

Moghazi, A.M.; Ali, K.A.; Wilde, S.A.; Zhou, Q.; Andersen, T.; Andresen, A.; Abu El-Enen, M.M., and Stern, R.J., 2012. Geochemistry, geochronology, and $\mathrm{Sr}-\mathrm{Nd}$ isotopes of the Late Neoproterozoic Wadi Kid volcano-sedimentary rocks, Southern Sinai, Egypt: implications for tectonic setting and crustal evolution. Lithos, 154, 47-165.

Moghazi, A.M.; Harbi, H.M., and Ali, K.A., 2011. Geochemistry of the Late Neoproterozoic Hadb adh Dayaheen ring complex, Central Arabian Shield: implications for the origin of raremetal-bearing post-orogenic A-type granites. J. Asian Earth Sciences ,42, 1324-1340.

Monecke,S.; Kuhnert, P.; Hotzel , H.; Slickers P., and Ehricht. P.,2007. Microarray based study on virulence- associated genes and resistance determinants of staphylococcus aurreus isolate from cattle. Vet.Microbiot,125,128-140

Monecke, T.; Kempe, U., and Götze, J., 2002. Genetic significance of the trace element content in metamorphic and hydrothermal quartz: A reconnaissance study: Earth and Planetary Science Letters, 202, 709-724

Moreno, J.A.; Molina, J.F.; Montero, P.; Abu Anbar, M.; Scarrow, J.H.; Cambeses, A., and Bea, F., 2014. Unraveling sources of A-type magmas in juvenile continental crust: Constraints from compositionally diverse Ediacaran post-collisional granitoids in the Katerina Ring Complex, southern Sinai, Egypt. Lithos ,192-195, 56-85

Moreno, J.A.; Montero, P.; Abu Anbar, M.; Molina, J.F.; Scarrow, J.H.; Talavera, C.; Cambeses, A., and Bea, F., 2012. SHRIMP U-Pb zircon dating of the Katerina Ring Complex: insights into the temporal sequence of Ediacaran calc-alkaline to peralkaline magmatism in southern Si- 
nai, Egypt. Gondwana Research, 21, 887-900.

Mushkin, A.; Navon, O.; Halicz, L.; Hartmann, G., and Stein, M., 2003. The petrogenesis of Atype magmas from the Amram Massif, southern Israel. J. Petrology, 44, 815-832.

Nagasawa, H., and Schnetzler, C.C., 1971. Partitiooning of rare earth, alkali and alkaline elements between phenocyrsts and acidic igneous magma. 35, 953-968.

Nasr,B.B., and El Sherbeni,H., 2001. Tectonic setting of gabal kilkbob granite,south eastern desert, Egypt. Annals Geol .Surv.Egypt, XXIV,115-129.

Nehlig, P.; Genna, A., and Asirfane, F., 2002. A review of the Pan-African evolution of the Shield. GeoArabia ,7, 103-124.

Oreskes, N., and Einaudi, M. T., 1990. Origin of rare earth-enriched hematite breccias at the Olympic Dam $\mathrm{Cu}-\mathrm{U}-\mathrm{Au}-\mathrm{ag}$ deposit, Roxby Downs, south Australia. Econ. Geol., 85, 1-28.

Panahi,A.; Young, G.M., and Rainibird, R.H., 2000. Behavior of major and trace elements (including REE ) during paleoproterozoic pedogenesis and digenetic alteration of an Archean granite near nile Marie, Quebec, Canada, Geochem. Cosmochem., Acta, G4(14),2199-2220.

Pearce, J.A., 1996. Sources and settings of granitic rocks. Inter. Geosci. New Magazine, 19 (4), 120-125.

Pearce, J.A.; Harris, N.B.W., and Tindle, A.G. 1984. Trace element discrimination Diagrams for the tectonic interpretation of granitic rocks. J. petrology, 25, 956-983.

Pollard, P. J.; Pichavant, M., and Charoy, B., 1987. Contrasting evolution of fluorine and boron-rich tin systems: Mineral. Deposita ,22, 315-321.

Raimbault, L.; Charoy, B.; Cuney, M., and Pollard, P. J. 1991. Comparative geochemistry of Ta-bearing granites. In: Source, Transport and deposition of Metals (Pagel, M., and Leroy, J., Eds.). Rotterdam: Balkema, 793-796.
Rapp, R.P., 1995. Amphibole out phase boundary in partially melted metabasalt, its control over liquid fraction and composition, and source permeability . J.Geol. Res., Solid Earth ,100, 15601-15610

Salvi, S., and Williams-Jones, A.E., 2005. Alkaline granite-syenite deposits. In: Rare-Element Geochemistry and Mineral Deposits(Linnen, R.L., \& Samson, I.M.,Eds.). Geol. Assoc. Canada Short Course Notes 17, 315-341.

Schnetzler, C.C., and Philopotts, J.A.,1970. geochemistry. Cosmochim.Acta, 34, 331-340.

Shand, S. J.,1951. Eruptive rocks. John Wiley, New York.

Schönenberger, J.; Köhler, J., and Markl, G., 2008. REE systematics of fluorides, calcite and siderite in peralkaline plutonic rocks from the Gardar Province, South Greenland. Chem. Geol., $247,16-35$

Stern, R.J., 1994. Arc assembly and continental collision in the Neoproterozoic East African Orogen: implications for the consolidation of Gondwanaland. Ann. Reviews of Earth and Planetary Sciences, 22, 319-351.

Stern, R.J., 2002. Crustal evolution in the East African Orogen: a Neodymium isotopic perspective. J. African Earth Sciences, 34, 109-117.

Stern, R.J., 2008. Neoproterozoic crustal growth: the solid Earth system during a critical episode of the Earth history. Gondwana Research, 14, $33-50$.

Stern, R.J., Johnson, P.R., 2010. Continental lithosphere pf the Arabian Plate: a geologic, petrologic, and geophysical synthesis. Earth Science Reviews, 101, 29-67.

Sun, S. S., and Nesbitt, R. W.,1978. Petrogenesis of Archean ultrabasic and basic volcanics: evidence from rare earth elements. Contrib. Minr. Petrol., 65, 301-328.

Takahashi, Y.; Shimizu, H.; Usui, A.; Kagi, H., and Normura, 2000. Direct observation of tetravalent cerium in ferromanganese nodules and 
crusts by X-ray absorption near-edge structure (XANES). Geochim cosmochim, Acta, 64, 2929-2935.

Turner, S.P.; Foden, J.D., and Morrison, R.S., 1992. Derivation of some A-type magmas by fractionation of basalticmagma - an example from the Padthaway Ridge, South Australia. Lithos, 28, 151-179.

Taylor, S.R., and McLennan , S.M.,1985. The continental crust : its composition and evolution. Blackwell, 312.

Vander Auwera, J.; Bogaerts, M.; Liégeois, J.P.; Demaiffe, D.; Wilmart, E.; Bolle, O., and Duchesne, J.C., 2003. Derivation of the 1.0-0.9 $\mathrm{Ga}$ ferro-potassic A-type granitoids of southern Norway by extreme differentiation from basic magmas. Precambrian Research, 124, 107-148.

Webster, J. D., and Holloway, J. R., 1988. Experimental constrains on the partitioning of $\mathrm{Cl}$ between topaz rhyolite melt and $\mathrm{H} 2 \mathrm{O}$ and $\mathrm{H} 2 \mathrm{O}+\mathrm{CO} 2$ fluids: New implications for granitic differentiation and ore deposition: Geochimica et Cosmochimica Acta, 52, 2091-2105

Webster, J.; Thomas, R.; Förster, H.J.; Seltmann, R., and Tappen, C., 2004. Geochemical evolution of halogen-enriched granite magmas and mineralizing fluids of the Zinnwald tin-tungsten mining district, Erzgebirge, Germany.
Mineralium Deposita, 39, 452-472.

Whalen, J. B.; Curri, K.L., and Chappell B.W., 1987. A-type granites geochemical characteristics, discrimination and petrogenesis. contributions to Mineralogy and petrology, 95, 407419.

Wilke, M., and Behrens, H., 1999. The dependence of the partitioning of iron and europium between plagioclase and hydrous tonalite melt on oxygen fugacity. Contrib. Mineral. Petrol., 137, $102-114$

ZhenHua; Wang Miao; Wang Xin-Qing; Zhu HaiBin; Lu Huan-Ming, and Y. Ando, 2002.Synthesis of Large Quantity Single-Walled Carbon Nanotubes by Arc Discharge, Chinese Physics Letters, 19, NO 1.

Zou, H., 1998. Trace element fractionation during modal and non modal dynamic melting and open-system melting: Amathematical treatment.Geochim cosmochim, Acta, 62, 711-717.

Zou, H., 2000. Modeling of trace element fractionation during non model dynamic melting with linear variations in mineral melt distribution coefficients. Geochim cosmochim, Acta, 64, 1095-1102.

Zou, H., and Zindler, A., 1996. Constraints on the degree of dynamic partial melting and source composition using concentration ratios in magmas. Geochim cosmochim, Acta, 60, 711-717.

$$
\begin{aligned}
& \text { جيولوجية وجيوكيميائية وإشعاعيه صخور الجرانيث بمنطقه وادى مره - جنوب }
\end{aligned}
$$

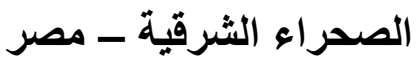

$$
\begin{aligned}
& \text { عادل الأفندى ، خيرية فوزى ، حسن عسران ، إيهاب أبو زيد و جيهان الثايب }
\end{aligned}
$$

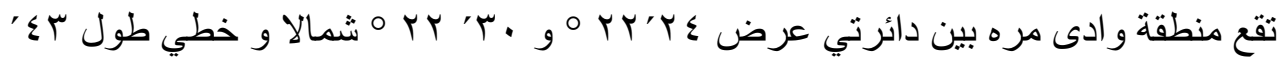

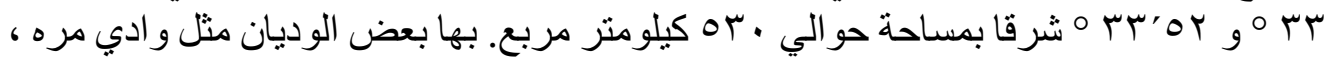

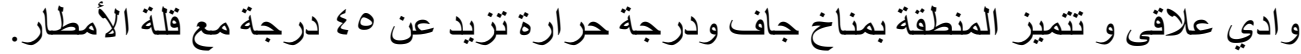

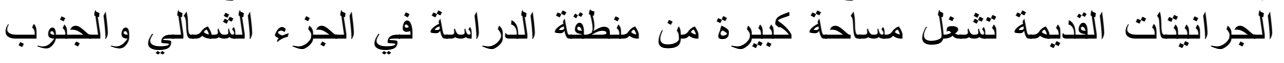

$$
\begin{aligned}
& \text { الثرقي من المنطقه وتنقسم الي كواتز ديوريت وصخر الجرانوديرويت وتتميز بغلبة الفلسبار }
\end{aligned}
$$

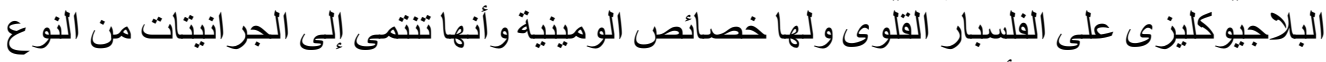

$$
\begin{aligned}
& \text { (A) - كالس قلوية نشأت فى بيئة قوس بركانى. }
\end{aligned}
$$


الجرانيتات الحديثة تقع من الثمال للجنوب حول جبل كالكبوب وتشئل جرانيت الفلسبار

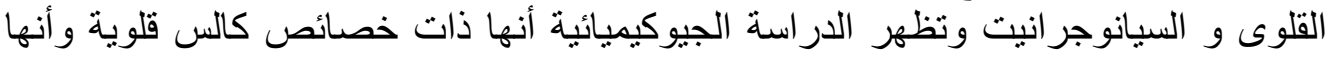

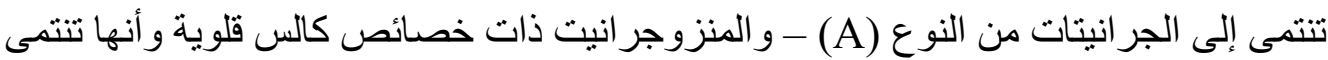

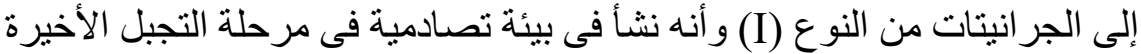

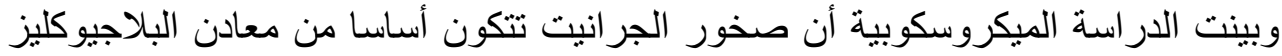

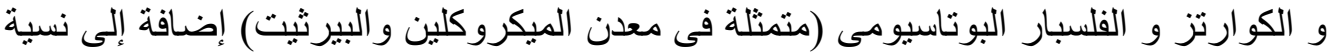

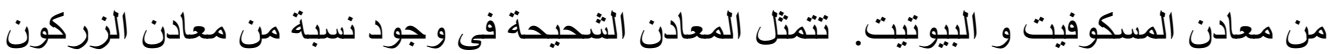

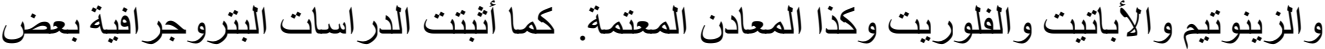

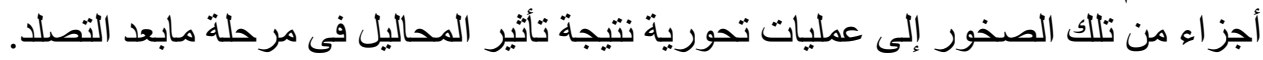

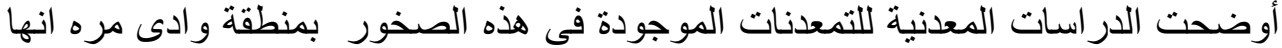

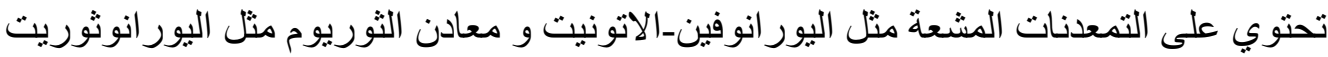
ومعادن النيوبيوم و التنتالم وذللك في صخورجر انيت الفلسبار القلوى و السيانوجر انيت و تحتوي

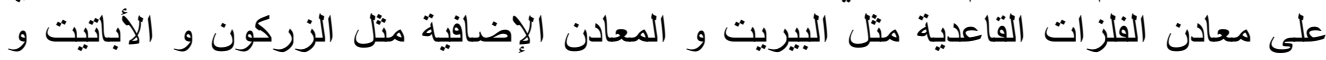

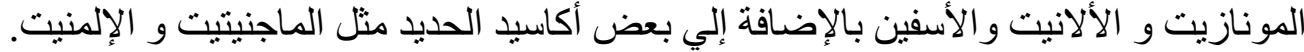

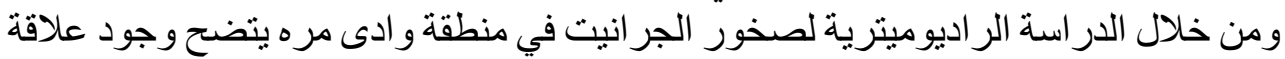

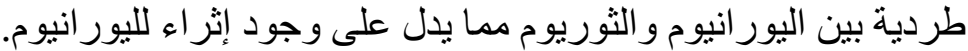

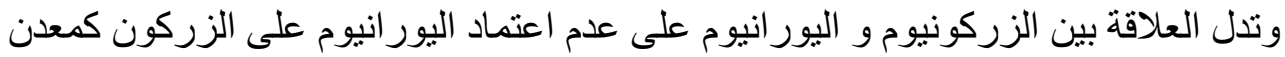

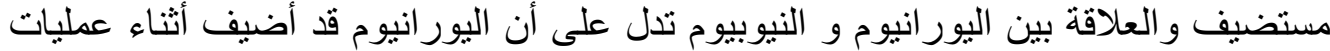

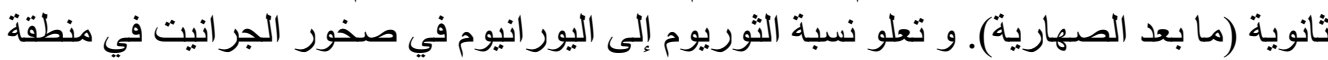

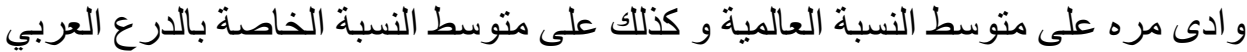

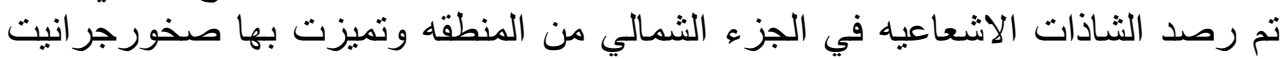

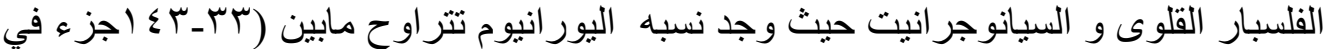

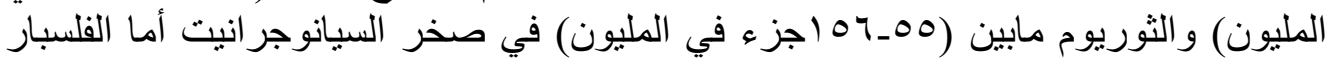

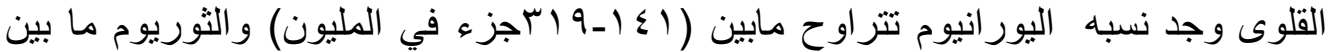

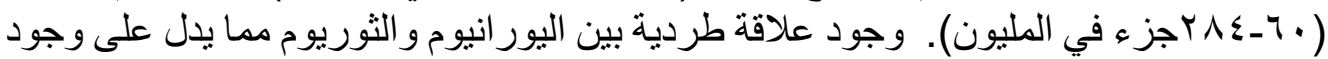
إثر اء لليور انيوم وتدل على أن اليور انيوم قد أضيف أثناء عمليات ثانوية (ما بعد الصهارية الصية). 